\title{
Synthesis of 2-ureido-4-ferrocenyl pyrimidine guests. Investigation of complementary molecular recognition of 2,6-diaminopyridine
}

\author{
Csaba Fehér, ${ }^{a}$ Máté Papp, ${ }^{\mathrm{a}}$ Ágnes Gömöry, ${ }^{\mathrm{b}}$ Lívia Nagy, ${ }^{\mathrm{c}}$ Johan Wouters, ${ }^{\mathrm{d}}$ György Lendvay, ${ }^{\mathrm{e}, \mathrm{f}}$ Rita \\ Skoda-Földes ${ }^{* a}$ \\ ${ }^{a}$ Institute of Chemistry, Department of Organic Chemistry, University of Pannonia, Egyetem u. 10. (P.O.Box 158) H- \\ 8200 Veszprém, Hungary \\ ${ }^{\mathrm{b}}$ Instrumentation Center, Research Centre for Natural Sciences, Hungarian Academy of Sciences, H-1117 Budapest, \\ Hungary \\ ${ }^{\mathrm{c} J a ́ n o s ~ S z e n t a ́ g o t h a i ~ R e s e a r c h ~ C e n t e r, ~ U n i v e r s i t y ~ o f ~ P e ́ c s, ~ I f j u ́ s a ́ g ~ u ́ t j a ~ 2 o, ~ H-7624 ~ P e ́ c s, ~ H u n g a r y ~}$ \\ ${ }^{\mathrm{d}}$ Department of Chemistry, University of Namur, B-500o Namur, Belgium \\ ${ }^{\mathrm{e}}$ Institute of Chemistry, Department of General and Inorganic Chemistry, University of Pannonia, H-820o \\ Veszprém, Hungary \\ ${ }^{\mathrm{f}}$ Institute of Materials and Environmental Chemistry, Research Centre for Natural Sciences, Hungarian Academy of \\ Sciences, H-1117 Budapest, Hungary
}

Supporting Information ABSTRACT: Novel 2-ureido-4-ferrocenylpyrimidines have been synthesized from iodoferrocene by a three-step reaction
sequence. XRD analysis and spectroscopic data support the presence of an intramolecular hydrogen bond between a ni-
trogen atom of the pyrimidine ring and an NH group of the urea moiety. Both theoretical calculations and NMR investi-
gations prove the existence of two isomeric forms in solution both of which can form hydrogen bonds with an appropriate
guest via an acceptor - donor - acceptor bonding pattern. Complexation with 2,6-diaminopyridine can be proved either
by NMR spectroscopy or by electrochemical measurements.

\section{INTRODUCTION}

Ferrocene and its derivatives are the most commonly used mediators in amperometric biosensors. ${ }^{1}$ In ferrocene-based chemosensors, binding of a molecular guest at an adjacent receptor site induces a shift in the redox potential of the ferrocene/ferrocenium couple. As an example, ferrocene-urea derivatives have been used as anion receptors ${ }^{2}$ in which the urea moiety contributed two relatively strong hydrogenbonding sites to the interaction. The voltammetric behavior of dendrimers with peripheral ferrocenyl-urea groups were found to be very sensitive to the presence of hydrogenphosphate anions at submillimolar concentration levels. ${ }^{3}$ Ureas obtained by the reaction of chiral ferrocenylamines with aryl isocyanates were used for the detection of chiral carboxylate ions. The selectivity in the complexation of these anions was high enough to allow opposite enantiomers to be distinguished by electrochemical means. ${ }^{4}$ Heteroditopic ferrocene-based ureas were designed as receptors for various anions and cations. ${ }^{5}$

Other applications of ferrocenylureas take advantage of the hydrophilicity of the urea group or its ability to form hydrogen bonds with neutral molecules. Palladium complexes of phosphinoferrocenes with a hydrophilic urea tag were demonstrated to catalyze efficiently the cross-coupling of boronic acids and acyl halides in a toluene/water biphasic mixture. ${ }^{6}$ Stable dimers of tetraurea calix[4]arenes with ferrocenyl moieties are formed due to multiple hydrogen bonds between the ureas on both calixarene heads. The dimers undergo dissociation upon oxidation of the ferrocene groups, so dimerization equilibrium can be controlled by the oxidation state of the compound. ${ }^{7}$

The ureido group itself is often part of structures forming multiple hydrogen-bonding arrays leading to the formation of supramolecular polymers ${ }^{8}$ or gels. ${ }^{9}$ The ureidopyrimidone functionality serves as a self-complementary quadruple hy- 
drogen-bonding unit in supramolecular assemblies. 6Methyl-2-butyl-ureidopyrimidone was shown to dimerize via four hydrogen bonds in the solid state as well as in $\mathrm{CHCl}_{3}$ solution via a donor-donor-acceptor-acceptor (DDAA) array of hydrogen-bonding sites. ${ }^{10}$ Complex formation of appropriate guests and 2-ureido- $4[1 \mathrm{H}]$-pyrimidinone moieties at $\mathrm{Au}$ surfaces was observed through quadruple hydrogen bonds, moreover, complexation could be conveniently controlled via changing solvent or temperature."

The introduction of the redox-active ferrocene moiety into these systems may make it possible to follow or even alter interactions by electrochemical methods. ${ }^{12}$ The solventinduced tautomerism of the dimeric $4[1 \mathrm{H}]$-pyrimidinone form of a ferrocene-substituted ureidopyrimidinone derivative to the monomeric $6[1 \mathrm{H}]$-pyrimidinone form could be followed by cyclic voltammetry. ${ }^{13}$ A photoinduced electron transfer through quadruple complementary hydrogen bonds was observed in a 2-ureido-4 $(1 \mathrm{H})$-pyrimidinone-bridged assembly, constructed with ferrocene and fullerene as the donor and acceptor, respectively. ${ }^{14}$ A remarkable level of electronic communication was observed between the two equivalent ferrocene centers of an ureido pyrimidinedione dimer in which the ferrocene is directly attached to the selfcomplementary DDAA hydrogen-bonding array. ${ }^{15}$ A drop of electronic communication of ferrocenyl moieties of ureidopyrimidinone dimers could be achieved by the addition of $\mathrm{CF}_{3} \mathrm{COOH}$, which induced dissociation of the $\mathrm{H}$ bonds. ${ }^{16}$ The strength of hydrogen-bonding interactions between ferrocenyl-urea and ferrocenyl-amidopyridine hosts and appropriate guests could be controlled electrochemically by oxidation of the ferrocene host or reduction of the guest moiety. ${ }^{17}$

As a continuation of our work concerning ferrocene labelled pyrimidine derivatives, ${ }^{18}$ in the present study we report on a three-step synthesis of new 2-ureido-4-ferrocenyl pyrimidines and investigation of their host-guest interactions with 2,6-diaminopyridine.

\section{EXPERIMENTAL SECTION}

General Comments. With the exception of the carbonylative Sonogashira coupling, all reactions were carried out under $\mathrm{Ar}$ using solvents which were dried by routine procedures. Starting materials (ferrocene, alkynes and isocyanates) and catalysts $\left(\mathrm{PdCl}_{2}\left(\mathrm{PPh}_{3}\right)_{2}, \mathrm{CuI}\right)$ were purchased from Aldrich and used without further purification. Iodoferrocene was prepared as described previously. ${ }^{19}$ Melting points were determined on a Kofler hot-plate apparatus (HMK Franz Küstner, Germany) and are uncorrected. ${ }^{1} \mathrm{H}$ and ${ }^{13} \mathrm{C}$ NMR spectra were recorded in $\mathrm{CDCl}_{3}$ on a Bruker Avance 400 spectrometer at $400 \mathrm{MHz}$ and $100 \mathrm{MHz}$, respectively. Chemical shifts $(\delta)$ are reported in ppm relative to $\mathrm{CHCl}_{3}$ (7.26 and 77.00 ppm for ${ }^{1} \mathrm{H}$ and ${ }^{13} \mathrm{C}$, respectively. Electrospray (ESI) mass spectra were obtained on a triple quadruple Micromass Quattro spectrometer (Waters, Milford, MA, USA). GC-MS measurements were carried out on a HP-5971A MSD connected to a HP-5890/II gas chromatograph. IR spectra were taken using a Thermo Nicolet Avatar 330 FT-IR instrument. Samples were prepared as $\mathrm{KBr}$ pellets. Elemental analyses were measured on a 1108 Carlo Erba apparatus.

General procedure for carbonylative Sonogashira coupling under homogeneous conditions. ${ }^{18 a}$ In a typical experiment a mixture of iodoferrocene (1) (o.5 mmol), $\mathrm{PdCl}_{2}\left(\mathrm{PPh}_{3}\right)_{2}$ (o.05 mmol), CuI (o.02 mmol), triethylamine (1 mmol), alkyne (2a-d) $(2.5 \mathrm{mmol})$ and THF $(5 \mathrm{ml})$ were transferred under an inert atmosphere into a stainless steel autoclave. It was charged with carbon monoxide ( 15 bar at room temperature) and heated with stirring in an oil bath at $60{ }^{\circ} \mathrm{C}$ for $12 \mathrm{~h}$. After cooling, the solvent was removed in vacuo. The products were isolated by column chromatography (silica gel, eluent: toluene). Carbonylative Sonogashira coupling with the supported catalyst A mixture of iodoferrocene (1) $(0.5 \mathrm{mmol})$, triethylamine $(1 \mathrm{mmol})$, $2 a(2.5 \mathrm{mmol})$ and THF $(7.5 \mathrm{ml})$ were transferred under an inert atmosphere into a stainless steel autoclave containing the SILPPd catalyst $60 \mathrm{mg}, 0.29 \mathrm{wt} \%$ of Pd on silica). ${ }^{20}$ It was charged with carbon monoxide (15 bar at room temperature) and heated with stirring in an oil bath at $60{ }^{\circ} \mathrm{C}$ for $12 \mathrm{~h}$. After cooling, the catalyst was filtered and reused without purification. The solvent was removed from the reaction mixture in vacuo. The products were isolated by column chromatography (silica gel, eluent: toluene).

${ }^{1} \mathrm{H}$ and ${ }^{13} \mathrm{C}$ NMR spectra of $3 \mathbf{a}$ and $3 \mathbf{b},{ }^{18 a} 3 \mathbf{c}^{21}$ and $3 \mathbf{d}^{18 a}$ are identical to those reported before.

1-Ferrocenyl-3-phenyl-2-propyn-1-one (3a). Yield: $91 \%$. Violet solid. Mp: 104-105 ${ }^{\circ} \mathrm{C}$. Anal. Calcd. for $\mathrm{C}_{19} \mathrm{H}_{14} \mathrm{FeO}$ : C, 72.64; $\mathrm{H}$, 4.49. Found: C, 72.81; H, 4.32.

1-Ferrocenyl-2-heptyn-1-one (3b). Yield: $65 \%$. Red oil. Anal. Calcd. for $\mathrm{C}_{17} \mathrm{H}_{18} \mathrm{FeO}$ : C, 69.41; H, 6.17. Found: $\mathrm{C}$, 69.22; $\mathrm{H}, 6.07$.

1-Ferrocenyl-2-hexyn-1-one (3c). Yield: $78 \%$. Red oil. Anal. Calcd. for $\mathrm{C}_{16} \mathrm{H}_{16} \mathrm{FeO}$ : C, 68.6o; H, 5.76. Found: C, 68.82; H, 5.87.

4,4-Dimethyl-1-ferrocenyl-2-pentyn-1-one (3d): Yield: $85 \%$. Red solid. Mp: 89-90 ${ }^{\circ} \mathrm{C}$. Anal. Calcd. for $\mathrm{C}_{17} \mathrm{H}_{18} \mathrm{FeO}$ : C, 69.41; H, 6.17. Found: C, 69.34; H, 6.32 .

Synthesis of 2-amino-4-ferrocenylpyrimidines. The 3substituted 1-ferrocenyl-2-propyn-1-one (3a-d) (0.2 mmol) was reacted with guanidine carbonate $(0.44 \mathrm{mmol})$ in $10 \mathrm{ml} \mathrm{EtOH}$ at reflux temperature for $12 \mathrm{~h}$. Evaporation of the solvent and column chromatography (silica gel, eluent: toluene:EtOAc=4:1) led to the products.

${ }^{1} \mathrm{H}$ and ${ }^{13} \mathrm{C}$ NMR spectra of $\mathbf{4} \mathbf{a}$ and $\mathbf{4} \mathbf{b}$ are identical to those reported before. ${ }^{18 a}$

2-Amino-4-ferrocenyl-6-phenylpyrimidine (4a). Yield: $54 \%$. Dark red solid. Mp: $159-160{ }^{\circ} \mathrm{C}$. Anal. Calcd. for $\mathrm{C}_{20} \mathrm{H}_{17} \mathrm{FeN}_{3}$ : C, $67.63 ; \mathrm{H}, 4.82$; N, 11.83 Found: C, 67.71; H, 4.75; N, 12.01 .

2-Amino-4-butyl-6-ferrocenylpyrimidine (4b). Yield: $85 \%$. Dark red solid. Mp: 114-115 ${ }^{\circ} \mathrm{C}$. Anal. Calcd. for $\mathrm{C}_{18} \mathrm{H}_{21} \mathrm{FeN}_{3}$ : C, 64.49; H, 6.31; N, 12.53. Found: C, 64.31; H, 6.51; N, 12.45.

2-Amino-4-ferrocenyl-6-propylpyrimidine (4c). Yield: $78 \%$. Red solid Mp: $120-123^{\circ} \mathrm{C} . \delta_{\mathrm{H}}\left(400 \mathrm{MHz}, \mathrm{CDCl}_{3}\right): 6.57$ (s, $\left.1 \mathrm{H}, 5-\mathrm{H}\right), 5.49$ (s, $\left.2 \mathrm{H}, \mathrm{NH}_{2}\right), 4.89$ (t, J=1.9 Hz, $\left.2 \mathrm{H}, \mathrm{Cp}\right), 4.40(\mathrm{t}, J=1.9 \mathrm{~Hz}, 2 \mathrm{H}, \mathrm{Cp})$, 4.06 (s, $5 \mathrm{H}, \mathrm{Cp}$ ), 2.53 (t, J=7.5 Hz, 2H, C6- $\mathrm{CH}_{2}$ ), 1.73 (sext, J=7.5 $\left.\mathrm{Hz}, 2 \mathrm{H}, 4-\mathrm{CH}_{2}-\mathrm{CH}_{2}\right), 0.99\left(\mathrm{t}, J=7.5 \mathrm{~Hz},{ }_{3} \mathrm{H}, \mathrm{CH}_{3}\right) . \delta_{\mathrm{C}}(100 \mathrm{MHz}$, $\left.\mathrm{CDCl}_{3}\right): 170.8,168.6,163.3,106.6,81.3,70.7,70.0,68.1,39.9,22.4$, 14.1. $v_{\max }\left(\mathrm{KBr} ; \mathrm{cm}^{-1}\right): 3477,3295,3152,2958,1633,1580,1545$. EI MS ( $\mathrm{m} / \mathrm{z}$ (relative intensity)) $321\left(\mathrm{M}^{+}, 100\right), 306$ (5), 293 (38), 256 (10), 240 (4), 228 (3), 227 (3), 212 (2), 187 (3), 153 (7), 147 (11), 146 (7), 145 (12), 121 (9), 89 (5), 56 (6). Anal. Calcd. for $\mathrm{C}_{17} \mathrm{H}_{19} \mathrm{FeN}_{3}$ : C, 63.57; H, 5.96; N, 13.08. Found: C, 63.49; H, 6.11; N, 13.21 .

2-Amino-4-tert-butyl-6-ferrocenylpyrimidine (4d). Yield: $67 \%$. Red solid Mp: $101-104{ }^{\circ} \mathrm{C} . \delta_{\mathrm{H}}\left(400 \mathrm{MHz}, \mathrm{CDCl}_{3}\right): 6.74(\mathrm{~s}, 1 \mathrm{H}, 5-\mathrm{H})$, $5.48\left(\mathrm{~s}, 2 \mathrm{H}, \mathrm{NH}_{2}\right), 4.91$ (t, J=1.9 Hz, $\left.2 \mathrm{H}, \mathrm{Cp}\right), 4.40(\mathrm{t}, J=1.9 \mathrm{~Hz}, 2 \mathrm{H}$, Cp), 4.07 (s, $5 \mathrm{H}, \mathrm{Cp}), 1.33\left(\mathrm{~s}, 9 \mathrm{H}, \mathrm{C}\left(\mathrm{CH}_{3}\right)_{3}\right) . \delta_{\mathrm{C}}\left(100 \mathrm{MHz}, \mathrm{CDCl}_{3}\right)$ : 178.2, 168.5, 163.2, 103.1, 82.0, 70.6, 70.1, 68.1, 37.2, 29.7. $v_{\max }(\mathrm{KBr}$; $\left.\mathrm{cm}^{-1}\right): 3455,3323,3190,2960,1633,1573,1536$. EI MS ( $\mathrm{m} / \mathrm{z}$ (relative intensity)) $335\left(\mathrm{M}^{+}, 100\right), 320$ (13), 293 (10), 254 (22), 160 (17), 145 (7), 121 (7), 89 (4), 56 (4). Anal. Calcd. for $\mathrm{C}_{18} \mathrm{H}_{21} \mathrm{FeN}_{3}$ : C, 64.49; $\mathrm{H}$, 6.31; N, 12.53. Found: C, 64.41; H, 6.57; N, 12.62.

Synthesis of 2-ureido-4-ferrocenylpyrimidines. The 2amino-4-ferrocenylpyrimidines (4a-d) $(0.2 \mathrm{mmol})$ and the corresponding isocyanate $(1.4 \mathrm{mmol})$ were heated under stirring in the absence of any solvent at $100{ }^{\circ} \mathrm{C}$. The progress of the reac- 
tions was monitored by TLC. The products were purified by column chromatography (silica gel, eluent: toluene:EtOAc=4:1).

1-(4-Ferrocenyl-6-phenylpyrimidin-2-yl)-3-phenylurea (5a). Yield: $77 \%$. Dark red solid. Mp: $188-191{ }^{\circ} \mathrm{C}$. $\delta_{\mathrm{H}}\left(400 \mathrm{MHz}, \mathrm{CDCl}_{3}\right)$ : $11.75(\mathrm{~s}, 1 \mathrm{H}, \mathrm{NH}), 8.04-8.09(\mathrm{~m}, 2 \mathrm{H}, \mathrm{Ph}), 7.70(\mathrm{~d}, J=7.7 \mathrm{~Hz}, 2 \mathrm{H}$, $\mathrm{Ph}), 7.55-7.58(\mathrm{~m}, 3 \mathrm{H}, \mathrm{Ph}), 7.53(\mathrm{~s}, 1 \mathrm{H}, \mathrm{NH}), 7.40\left(\mathrm{t}, J=7.7 \mathrm{~Hz},{ }_{2} \mathrm{H}\right.$, $\mathrm{Ph}), 7.35(\mathrm{~s}, 1 \mathrm{H}, 5-\mathrm{H}), 7.13(\mathrm{t}, J=7.7 \mathrm{~Hz}, 1 \mathrm{H}, \mathrm{Ph}), 5.03(\mathrm{t}, J=1.6 \mathrm{~Hz}$, $2 \mathrm{H}, \mathrm{Cp}), 4.60(\mathrm{t}, J=1.6 \mathrm{~Hz}, 2 \mathrm{H}, \mathrm{Cp}), 4.13(\mathrm{~s}, 5 \mathrm{H}, \mathrm{Cp}) . \delta_{\mathrm{C}}(100 \mathrm{MHz}$, $\left.\mathrm{CDCl}_{3}\right): 170.1,164.4,157.9,152.2,138.7,136.8,131.3,129.3,129.2$, 127.3, 123.7, 120.0, 106.6, 79.9, 71.8, 70.4, 68.4. $v_{\max }\left(\mathrm{KBr} ; \mathrm{cm}^{-1}\right)$ : 3434, 3207, 1693, 1581, 1531. ESI MS ( $\mathrm{m} / \mathrm{z}$ (relative intensity)) 474 $\left(\mathrm{M}^{+}, 75\right), 475\left([\mathrm{M}+\mathrm{H}]^{+}, 58\right), 497\left([\mathrm{M}+\mathrm{Na}]^{+}, 100\right), 971\left([2 \mathrm{M}+\mathrm{Na}]^{+}\right.$, 22). Mass required for $\mathrm{C}_{27} \mathrm{H}_{22} \mathrm{FeN}_{4} \mathrm{O}: 474$, found: 474.. Anal. Calcd. for $\mathrm{C}_{27} \mathrm{H}_{22} \mathrm{FeN}_{4} \mathrm{O}: \mathrm{C}, 68.37 ; \mathrm{H}, 4.67 ; \mathrm{N}, 11.81$. Found: $\mathrm{C}$, 68.14; H, 4.72; N, 11.89 .

1-Butyl-3-(4-ferrocenyl-6-phenylpyrimidin-2-yl)-urea

(5)

Yield: $68 \%$. Red oil. $\delta_{\mathrm{H}}\left(400 \mathrm{MHz}, \mathrm{CDCl}_{3}\right): 9.37\left(\mathrm{t}, J=5.3 \mathrm{~Hz},{ }_{1} \mathrm{H}\right.$, $\mathrm{NH}), 7.98-8.01(\mathrm{~m}, 2 \mathrm{H}, \mathrm{Ph}), 7.51-7.54\left(\mathrm{~m},{ }_{3} \mathrm{H}, \mathrm{Ph}\right), 7.46(\mathrm{~s}, 1 \mathrm{H}$, $\mathrm{NH}), 7.28(\mathrm{~s}, 1 \mathrm{H}, 5-\mathrm{H}), 4.94(\mathrm{t}, J=1.9 \mathrm{~Hz}, 2 \mathrm{H}, \mathrm{Cp}), 4.54(\mathrm{t}, J=1.9 \mathrm{~Hz}$, $\left.{ }_{2} \mathrm{H}, \mathrm{Cp}\right), 4.10(\mathrm{~s}, 5 \mathrm{H}, \mathrm{Cp}), 3.47\left(\mathrm{td}, J=6.9 \mathrm{~Hz}, J=5.3 \mathrm{~Hz}, 2 \mathrm{H}, \mathrm{CH}_{2}-\right.$ $\mathrm{NH}), 1.66-1.73\left(\mathrm{~m}, 2 \mathrm{H}, \mathrm{CH}_{2}\right), 1.47-1.56\left(\mathrm{~m}, 2 \mathrm{H}, \mathrm{CH}_{2}\right), 1.00(\mathrm{t}, J=7.3$ $\left.\mathrm{Hz}, 3 \mathrm{H}, \mathrm{CH}_{3}\right) . \delta_{\mathrm{C}}\left(100 \mathrm{MHz}, \mathrm{CDCl}_{3}\right): 169.7,164.3,158.3,154.8$, 137.0, 131.1, 129.1, 127.2, 106.2, 80.1, 71.5, 70.3, 68.2, 39.9, 32.2, 20.5, 14.O. $v_{\max }\left(\mathrm{KBr} ; \mathrm{cm}^{-1}\right)$ : 3434, 3233, 1682, 1580, 1525. Mass Calcd. for $\mathrm{C}_{25} \mathrm{H}_{26} \mathrm{FeN}_{4} \mathrm{O}: 454.15$. ESI MS ( $\mathrm{m} / \mathrm{z}$ (relative intensity)) $454\left(\mathrm{M}^{+}\right.$, $32), 455\left([\mathrm{M}+\mathrm{H}]^{+}, 36\right), 477\left([\mathrm{M}+\mathrm{Na}]^{+}, 100\right), 931\left([2 \mathrm{M}+\mathrm{Na}]^{+}, 28\right)$. Anal. Calcd. for $\mathrm{C}_{25} \mathrm{H}_{26} \mathrm{FeN}_{4} \mathrm{O}: \mathrm{C}, 66.09 ; \mathrm{H}, 5.77 ; \mathrm{N}, 12.33$. Found: C, 66.o1; H, 5.91; N, 12.17 .

1-Cyclohexyl-3-(4-ferrocenyl-6-phenylpyrimidin-2-yl)-urea (5c). Yield: $67 \%$. Red solid. Mp: $135-139{ }^{\circ} \mathrm{C}$. $\delta_{\mathrm{H}}\left(400 \mathrm{MHz}, \mathrm{CDCl}_{3}\right): 9.35$ (d, J=7.6 Hz, $1 \mathrm{H}, \mathrm{NH}), 7.99-8.05(\mathrm{~m}, 2 \mathrm{H}, \mathrm{Ph}), 7.52-7.56(\mathrm{~m}, 3 \mathrm{H}$, $\mathrm{Ph}), 7.38(\mathrm{~s}, 1 \mathrm{H}, \mathrm{NH}), 7.29(\mathrm{~s}, 1 \mathrm{H}, 5-\mathrm{H}), 4.96(\mathrm{t}, J=1.9 \mathrm{~Hz} 2 \mathrm{H}, \mathrm{Cp})$, $4.56(\mathrm{t}, J=1.9 \mathrm{~Hz}, 2 \mathrm{H}, \mathrm{Cp}), 4.12(\mathrm{~s}, 5 \mathrm{H}, \mathrm{Cp}), 3.81-3.91\left(\mathrm{~m},{ }_{1} \mathrm{H}, \mathrm{CH}\right)$, 2.11-2.21 (m, $2 \mathrm{H}$, cHex), 1.8o-1.86 (m, $2 \mathrm{H}, \mathrm{cHex}), 1.67-1.71(\mathrm{~m}, 1 \mathrm{H}$, cHex), 1.23-1.52 (m, $5 \mathrm{H}, \mathrm{cHex}) . \delta_{\mathrm{C}}\left(100 \mathrm{MHz}, \mathrm{CDCl}_{3}\right): 169.6,164.4$, 158.4, 154.0, 137.0, 131.1, 129.1, 127.2, 106.1, 80.2, 71.6, 70.3, 68.2, 49.1, 34.0, 26.o, 25.2. $v_{\max }\left(\mathrm{KBr} ; \mathrm{cm}^{-1}\right): 3436,3236,1681,1578,1527$. ESI MS $\left(m / z\right.$ (relative intensity)) $480\left(\mathrm{M}^{+}, 49\right), 481\left([\mathrm{M}+\mathrm{H}]^{+}, 22\right)$, $503\left([\mathrm{M}+\mathrm{Na}]^{+}, 100\right), 983\left([2 \mathrm{M}+\mathrm{Na}]^{+}, 37\right)$. Mass required for $\mathrm{C}_{27} \mathrm{H}_{28} \mathrm{FeN}_{4} \mathrm{O}: 480$, found: 480 . Anal. Calcd. for $\mathrm{C}_{27} \mathrm{H}_{28} \mathrm{FeN}_{4} \mathrm{O}: \mathrm{C}$, 67.51; H, 5.87; N, 11.66. Found: C, 67.32; H, 5.82; N, 11.81 .

1-(3-Chloropropyl)-3-(4-ferrocenyl-6-phenylpyrimidin-2-yl)-urea (5d). Yield: $97 \%$. Red solid. Mp: $42-44{ }^{\circ} \mathrm{C}$. $\delta_{\mathrm{H}}\left(400 \mathrm{MHz}, \mathrm{CDCl}_{3}\right)$ : $9.57(\mathrm{t}, J=5.9 \mathrm{~Hz}, 1 \mathrm{H}, \mathrm{NH}), 7.98-8.03(\mathrm{~m}, 2 \mathrm{H}, \mathrm{Ph}), 7.52-7.55(\mathrm{~m}$, $3 \mathrm{H}, \mathrm{Ph}), 7.48(\mathrm{~s}, 1 \mathrm{H}, \mathrm{NH}), 7.30(\mathrm{~s}, 1 \mathrm{H}, 5-\mathrm{H}), 4.96(\mathrm{t}, J=1.9 \mathrm{~Hz}, 2 \mathrm{H}$, Cp), $4.55(\mathrm{t}, J=1.9 \mathrm{~Hz}, 2 \mathrm{H}, \mathrm{Cp}), 4.11(\mathrm{~s}, 5 \mathrm{H}, \mathrm{Cp}), 3.73(\mathrm{t}, J=6.3 \mathrm{~Hz}$, $\left.2 \mathrm{H}, \mathrm{Cl}-\mathrm{C}_{2}\right), 3.64\left(\mathrm{td}, J=6.3 \mathrm{~Hz}, J=5.9 \mathrm{~Hz}, 2 \mathrm{H}, \mathrm{NH}-\mathrm{C}_{2}\right), 2.16$ (qui, J=6.3 Hz, $2 \mathrm{H}, \mathrm{CH}_{2}-\mathrm{CH}_{2}-\mathrm{Cl}$ ). $\delta_{\mathrm{C}}\left(100 \mathrm{MHz}, \mathrm{CDCl}_{3}\right): 169.8$, $164.3,158.2$, 155.0, 136.8, 131.2, 129.2, 127.2, 106.3, 80.0, 71.7, 70.3, 68.3, 42.7, 37.1, 32.6. $v_{\max }\left(\mathrm{KBr} ; \mathrm{cm}^{-1}\right): 3438,3246,1682,1590,1580$, 1526. ESI MS ( $\mathrm{m} / \mathrm{z}$ (relative intensity) $474\left(\mathrm{M}^{+}, 45\right), 475\left([\mathrm{M}+\mathrm{H}]^{+}\right.$, $38), 497\left([\mathrm{M}+\mathrm{Na}]^{+}, 100\right), 971\left([2 \mathrm{M}+\mathrm{Na}]^{+}, 8\right)$. Mass required for $\mathrm{C}_{24} \mathrm{H}_{23} \mathrm{ClFeN}_{4} \mathrm{O}: 474$, found: 474. Anal. Calcd. for $\mathrm{C}_{24} \mathrm{H}_{23} \mathrm{ClFeN}_{4} \mathrm{O}$ : C, 60.72; H, 4.88; N, 11.8o. Found: C, 60.98; H, 4.78; N, 12.01.

1-(4-Butyl-6-ferrocenylpyrimidin-2-yl)-3-phenylurea (5e). Yield: $99 \%$. Dark red solid. Mp: $146-148^{\circ} \mathrm{C}$. $\delta_{\mathrm{H}}\left(400 \mathrm{MHz}, \mathrm{CDCl}_{3}\right): 11.72$ (s, $1 \mathrm{H}, \mathrm{NH}), 7.66(\mathrm{dd}, J=7.7 \mathrm{~Hz}, J=1.1 \mathrm{~Hz}, 2 \mathrm{H}, \mathrm{Ph}), 7.36-7.41(\mathrm{~m}$, $3 \mathrm{H}, \mathrm{Ph}, \mathrm{NH}), 7.11$ (tt, J=7.7 Hz, J=1.1 Hz, $1 \mathrm{H}, \mathrm{Ph}), 6.81(\mathrm{~s}, 1 \mathrm{H}, 5-\mathrm{H})$, $4.94(\mathrm{t}, J=1.9 \mathrm{~Hz}, 2 \mathrm{H}, \mathrm{Cp}), 4.54(\mathrm{t}, J=1.9 \mathrm{~Hz}, 2 \mathrm{H}, \mathrm{Cp}), 4.10\left(\mathrm{~s},{ }_{5} \mathrm{H}\right.$, Cp), $2.71\left(\mathrm{t}, J=7.4 \mathrm{~Hz}, 2 \mathrm{H}, \mathrm{C}_{4}-\mathrm{CH}_{2}\right.$ ), 1.78 (qui, $J=7.4 \mathrm{~Hz}, 2 \mathrm{H}, \mathrm{CH}_{2}$ ), 1.46 (sext, $\left.J=7.4 \mathrm{~Hz}, 2 \mathrm{H}, \mathrm{CH}_{2}\right), 0.99\left(\mathrm{t}, J=7.4 \mathrm{~Hz}, 3 \mathrm{H}, \mathrm{CH}_{3}\right) . \delta_{\mathrm{C}}(100$ $\left.\mathrm{MHz}, \mathrm{CDCl}_{3}\right): 170.5,168.8,157.2,152.2,138.3,129.0,123.4,119.8$, 109.4, 79.4, 71.4, 70.1, 68.0, 37.3, 30.6, 22.3, 13.8. $v_{\max }\left(\mathrm{KBr} ; \mathrm{cm}^{-1}\right)$ : $3438,3215,3166,3101,1690,1590,1537$. ESI MS ( $\mathrm{m} / \mathrm{z}$ (relative intensity) $455\left([\mathrm{M}+\mathrm{H}]^{+}, 57\right), 477\left([\mathrm{M}+\mathrm{Na}]^{+}, 100\right), 931\left([2 \mathrm{M}+\mathrm{Na}]^{+}\right.$, 21). Mass required for $\mathrm{C}_{25} \mathrm{H}_{27} \mathrm{FeN}_{4} \mathrm{O}$ : 455, found:455. Anal. Calcd. for $\mathrm{C}_{25} \mathrm{H}_{26} \mathrm{FeN}_{4} \mathrm{O}$ : C, 66.09; $\mathrm{H}, 5.77 ; \mathrm{N}, 12.33$. Found: $\mathrm{C}, 66.31 ; \mathrm{H}$, $5.61 ; \mathrm{N}, 12.41$.

1-Butyl-3-(4-butyl-6-ferrocenylpyrimidin-2-yl)-urea (5f). Yield: $83 \%$. Red solid. Mp: $45-47^{\circ} \mathrm{C}$. $\delta_{\mathrm{H}}\left(400 \mathrm{MHz}, \mathrm{CDCl}_{3}\right): 9.34(\mathrm{t}, J=$ $5.5 \mathrm{~Hz} \mathrm{1H}, \mathrm{NH}), 7.20(\mathrm{~s}, 1 \mathrm{H}, \mathrm{NH}), 6.74(\mathrm{~s}, 1 \mathrm{H}, 5-\mathrm{H}), 4.87(\mathrm{t}, J=1.9$ $\mathrm{Hz}, 2 \mathrm{H}, \mathrm{Cp}), 4.49$ (t, J= $1.9 \mathrm{~Hz}, 2 \mathrm{H}, \mathrm{Cp}), 4.07$ (s, $5 \mathrm{H}, \mathrm{Cp}), 3.43$ (ddd, J=6.9 Hz, J=5.7 Hz, J=5.5 Hz, $\left.2 \mathrm{H}, \mathrm{NH}-\underline{\mathrm{H}}_{2}\right), 2.64(\mathrm{t}, J=7.6$ $\left.\mathrm{Hz}, 2 \mathrm{H}, \mathrm{C}_{4}-\mathrm{CH}_{2}\right), 1.62-1.75\left(\mathrm{~m}, 4 \mathrm{H}, \mathrm{CH}_{2}\right), 1.46-1.54\left(\mathrm{~m}, 2 \mathrm{H}, \mathrm{CH}_{2}\right)$, 1.36-1.45 (m, $\left.2 \mathrm{H}, \mathrm{CH}_{2}\right), 0.99\left(\mathrm{t}, J=7.3 \mathrm{~Hz},{ }_{3} \mathrm{H}, \mathrm{CH}_{3}\right), 0.97(\mathrm{t}, J=7.3$ $\left.\mathrm{Hz},{ }_{3} \mathrm{H}, \mathrm{CH}_{3}\right) . \delta_{\mathrm{C}}\left(100 \mathrm{MHz}, \mathrm{CDCl}_{3}\right): 170.5,168.3,157.6,155.0$, 108.9, 79.7, 71.1, 69.9, 67.9, 39.6, 37.2, 31.9, 30.5, 22.2, 20.2, 13.8, 13.7. $v_{\max }\left(\mathrm{KBr} ; \mathrm{cm}^{-1}\right): 3443,3235,3089,1685,1590,1534$. ESI MS $(\mathrm{m} / \mathrm{z}$ (relative intensity) $) 435\left([\mathrm{M}+\mathrm{H}]^{+}, 31\right), 457\left([\mathrm{M}+\mathrm{Na}]^{+}, 100\right)$, $891\left([2 \mathrm{M}+\mathrm{Na}]^{+}, 27\right)$. Mass required for $\mathrm{C}_{23} \mathrm{H}_{31} \mathrm{FeN}_{4} \mathrm{O}: 435$, found: 435. Anal. Calcd. for $\mathrm{C}_{23} \mathrm{H}_{30} \mathrm{FeN}_{4} \mathrm{O}: \mathrm{C}, 63.60 ; \mathrm{H}, 6.96$; N, 12.90. Found: C, 63.51; H, 7.14; N, 12.78 .

1-(4-Butyl-6-ferrocenylpyrimidin-2-yl)-3-cyclohexylurea (5g). Yield: $84 \%$. Red solid. Mp: $50-52{ }^{\circ} \mathrm{C}$. $\delta_{\mathrm{H}}\left(400 \mathrm{MHz} \mathrm{CDCl}_{3}\right): 9.34$ (d, J=7.7 Hz, $1 \mathrm{H}, \mathrm{NH}), 7.15(\mathrm{~s}, 1 \mathrm{H}, \mathrm{NH}), 6.73(\mathrm{~s}, 1 \mathrm{H}, 5-\mathrm{H}), 4.87(\mathrm{t}$, $J=1.9 \mathrm{~Hz}, 2 \mathrm{H}, \mathrm{Cp}), 4.50(\mathrm{t}, J=1.9 \mathrm{~Hz}, 2 \mathrm{H}, \mathrm{Cp}), 4.08(\mathrm{~s}, 5 \mathrm{H}, \mathrm{Cp})$, 3.78-3.88 (m, $1 \mathrm{H}, \mathrm{cHex}), 2.64\left(\mathrm{t}, J=7.6 \mathrm{~Hz}, 2 \mathrm{H}, \mathrm{C}_{4}-\mathrm{CH}_{2}\right), 2.04-2.16$ (m, $2 \mathrm{H}, \mathrm{cHex}), 1.66-1.86\left(\mathrm{~m},{ }_{5} \mathrm{H}, \mathrm{cHex}, \mathrm{C}_{4}-\mathrm{CH}_{2}-\mathrm{C}_{2}\right)$, 1.26-1.51 (m, $7 \mathrm{H}$, cHex, $\left.\mathrm{CH}_{2} \mathrm{CH}_{3}\right), 0.97\left(\mathrm{t}, J=7.6 \mathrm{~Hz}, 3 \mathrm{H}, \mathrm{CH}_{3}\right) . \delta_{\mathrm{C}}(100 \mathrm{MHz}$, $\left.\mathrm{CDCl}_{3}\right): 170.5,168.2,157.7,154.4,108.8,79.7,71.1,79.9,67.8,48.5$, 37.2, 33.6, 30.5, 25.6, 24.7, 22.2, 13.8. $v_{\max }\left(\mathrm{KBr} ; \mathrm{cm}^{-1}\right): 3438,3219$, $1683,1588,1533$. ESI MS $\left(\mathrm{m} / \mathrm{z}\right.$ (relative intensity)) $461\left([\mathrm{M}+\mathrm{H}]^{+}\right.$, $60), 483\left([\mathrm{M}+\mathrm{Na}]^{+}, 100\right), 943\left([2 \mathrm{M}+\mathrm{Na}]^{+}, 22\right)$. Mass required for $\mathrm{C}_{25} \mathrm{H}_{33} \mathrm{FeN}_{4} \mathrm{O}: 461$, found: 461. Anal. Calcd. for $\mathrm{C}_{25} \mathrm{H}_{32} \mathrm{FeN}_{4} \mathrm{O}: \mathrm{C}$, 65.22; H, 7.01; N, 12.17. Found: C, 65.44; H, 6.95; N, 12.05.

1-(4-Butyl-6-ferrocenylpyrimidin-2-yl)-3-(3-chloropropyl)urea (5h). Yield: $94 \%$. Red oil. $\delta_{\mathrm{H}}\left(400 \mathrm{MHz}, \mathrm{CDCl}_{3}\right): 9.54(\mathrm{t}, J=5.6 \mathrm{~Hz}$, $\left.{ }_{1} \mathrm{H}, \mathrm{NH}\right), 7.30(\mathrm{~s}, 1 \mathrm{H}, \mathrm{NH}), 6.75(\mathrm{~s}, 1 \mathrm{H}, 5-\mathrm{H}), 4.88(\mathrm{t}, J=1.9 \mathrm{~Hz}, 2 \mathrm{H}$, $\mathrm{Cp}), 4.50(\mathrm{t}, \mathrm{J}=1.8 \mathrm{~Hz}, 2 \mathrm{H}, \mathrm{Cp}), 4.08\left(\mathrm{~s},{ }_{5} \mathrm{H}, \mathrm{Cp}\right), 3.70(\mathrm{t}, J=6.4 \mathrm{~Hz}$, $2 \mathrm{H}, \mathrm{Cl}-\mathrm{CH}_{2}$ ), 3.61 (qua, $\left.J=6.4 \mathrm{~Hz}, 2 \mathrm{H}, \mathrm{NH}-\mathrm{CH}_{2}\right), 2.65(\mathrm{t}, J=7.4 \mathrm{~Hz}$, $2 \mathrm{H}, \mathrm{C}_{4}-\mathrm{CH}_{2}$ ), 2.13 (qui, $J=6.4 \mathrm{~Hz}, 2 \mathrm{H}, \mathrm{CH}_{2}-\mathrm{CH}_{2}-\mathrm{Cl}$ ), 1.66-1.76 (m, $2 \mathrm{H}, \mathrm{C}_{4}-\mathrm{CH}_{2}-\mathrm{CH}_{2}$ ), 1.41 (sext, J=7.4 Hz, $2 \mathrm{H}, \mathrm{C}_{2}-\mathrm{CH}_{3}$ ), $0.97(\mathrm{t}$, $\left.J=7.4 \mathrm{~Hz},{ }_{3} \mathrm{H}, \mathrm{CH}_{3}\right) . \delta_{\mathrm{C}}\left(100 \mathrm{MHz}, \mathrm{CDCl}_{3}\right): 170.5,168.5,157.5,155.2$, 109.0, 79.5, 71.2, 79.9, 67.9, 42.5, 37.2, 36.9, 32.3, 30.5, 22.2, 13.8. $v_{\max }\left(\mathrm{KBr} ; \mathrm{cm}^{-1}\right): 3438,3235,1695,1684,1590,1533$. ESI MS $(\mathrm{m} / \mathrm{z}$ (relative intensity)) $455\left([\mathrm{M}+\mathrm{H}]^{+}, 53\right), 477\left([\mathrm{M}+\mathrm{Na}]^{+}, 100\right), 931$ $\left([2 \mathrm{M}+\mathrm{Na}]^{+}, 2 \mathrm{O}\right)$ Mass required for $\mathrm{C}_{22} \mathrm{H}_{28} \mathrm{ClFeN}_{4} \mathrm{O}: 455$, found: 455. Anal. Calcd. for $\mathrm{C}_{22} \mathrm{H}_{27} \mathrm{ClFeN}_{4} \mathrm{O}$ : $\mathrm{C}, 58.10 ; \mathrm{H}, 5.98 ; \mathrm{N}, 12.32$.. Found: C, $58.29 ; \mathrm{H}, 6.11 ; \mathrm{N}, 12.35$.

1-(4-Ferrocenyl-6-propylpyrimidin-2-yl)-3-phenylurea

Yield: $99 \%$. Dark red solid. Mp: $108-110^{\circ} \mathrm{C} . \delta_{\mathrm{H}}\left(400 \mathrm{MHz}, \mathrm{CDCl}_{3}\right)$ : $11.71(\mathrm{~s}, 1 \mathrm{H}, \mathrm{NH}), 7.66$ (d, J=7.5 Hz, 2H, Ph), 7.43 (s, $1 \mathrm{H}, \mathrm{NH}), 7.36-$ $7.41(\mathrm{~m}, 2 \mathrm{H}, \mathrm{Ph}), 7.11(\mathrm{tt}, J=7.5 \mathrm{~Hz}, J=1.1 \mathrm{~Hz}, 1 \mathrm{H}, \mathrm{Ph}), 6.81\left(\mathrm{~s}, 1 \mathrm{H}, 5^{-}\right.$ H), 4.94 (t, J=1.9 Hz, 2H, Cp), 4.54 (t, J=1,9 Hz, 2H, Cp), 4.10 (s, ${ }_{5} \mathrm{H}, \mathrm{Cp}$ ), $2.69\left(\mathrm{t}, J=7.5 \mathrm{~Hz}, 2 \mathrm{H}, \mathrm{C} 6-\mathrm{CH}_{2}\right), 1.83$ (sext, J=7.5 Hz, $2 \mathrm{H}$, $\left.4-\mathrm{CH}_{2}-\mathrm{CH}_{2}\right), 1.04\left(\mathrm{t}, J=7.5 \mathrm{~Hz},{ }_{3} \mathrm{H}, \mathrm{CH}_{3}\right) . \delta_{\mathrm{C}}\left(100 \mathrm{MHz}, \mathrm{CDCl}_{3}\right)$ : $170.5,168.9,157.2,152.3,138.3,129.0,123.5,119.9,109.6,79.5,71.5$, $70.2,68.2,39.5,21.8,13.8 . v_{\max }\left(\mathrm{KBr} ; \mathrm{cm}^{-1}\right): 3102,1683,1599,1588$, 1538. ESI MS $\left(m / z\right.$ (relative intensity)) $441\left([\mathrm{M}+\mathrm{H}]^{+}, 52\right), 463$ $\left([\mathrm{M}+\mathrm{Na}]^{+}, 100\right), 903\left([2 \mathrm{M}+\mathrm{Na}]^{+}, 30\right)$. Mass required for $\mathrm{C}_{24} \mathrm{H}_{25} \mathrm{FeN}_{4} \mathrm{O}: 441$, found: 441. Anal. Calcd. for $\mathrm{C}_{24} \mathrm{H}_{24} \mathrm{FeN}_{4} \mathrm{O}: \mathrm{C}$, 65.47; H, 5.49; N, 12.72. Found: C, 65.21; H, 5.45; N, 12.81 .

1-Butyl-3-(4-ferrocenyl-6-propylpyrimidin-2-yl)-urea (5j). Yield: $82 \%$. Red oil. $\delta_{\mathrm{H}}\left(400 \mathrm{MHz}, \mathrm{CDCl}_{3}\right): 9.34$ (brs, $\left.1 \mathrm{H}, \mathrm{NH}\right), 7.22$ (s, $\left.{ }_{1} \mathrm{H}, \mathrm{NH}\right), 6.74$ (s, $\left.1 \mathrm{H}, 5-\mathrm{H}\right), 4.86$ (brs, $2 \mathrm{H}, \mathrm{Cp}$ ), 4.49 (brs, $2 \mathrm{H}, \mathrm{Cp}$ ), $4.07(\mathrm{~s}, 5 \mathrm{H}, \mathrm{Cp}), 3.38-3.47\left(\mathrm{~m}, 2 \mathrm{H}, \mathrm{NH}-\mathrm{CH}_{2}\right), 2.62(\mathrm{t}, J=7.4 \mathrm{~Hz}$, $2 \mathrm{H}, \mathrm{C} 6-\mathrm{CH}_{2}$ ), 1.76 (sext, J=7.6 Hz, $2 \mathrm{H}, \mathrm{C} 6-\mathrm{CH}_{2} \mathrm{CH}_{2}$ ), 1.65 (qui, $J=7.2 \mathrm{~Hz}, 2 \mathrm{H}, \mathrm{NH}-\mathrm{CH}_{2} \mathrm{CH}_{2}$ ), 1.49 (sext, J=7.2 Hz, $2 \mathrm{H}, \mathrm{NH}-$ $\mathrm{CH}_{2} \mathrm{CH}_{2} \mathrm{CH}_{2}$ ), 0.94-1.05 (m, 6H, $\left.\mathrm{CH}_{3}\right) . \delta_{\mathrm{C}}\left(100 \mathrm{MHz}, \mathrm{CDCl}_{3}\right)$ : 170.2, 168.4, 157.7, 155.1, 109.0, 79.7, 71.1, 70.0, 67.9, 39.5, 39.4, 31.9, 21.7, 20.2, $13.7(2 \mathrm{C}) . v_{\max }\left(\mathrm{KBr} ; \mathrm{cm}^{-1}\right): 3447,3236,1687,1591,1534 . \mathrm{ESI}$ MS ( $\mathrm{m} / \mathrm{z}$ (relative intensity)) $421\left([\mathrm{M}+\mathrm{H}]^{+}, 32\right), 443\left([\mathrm{M}+\mathrm{Na}]^{+}\right.$, 100), $863\left([2 \mathrm{M}+\mathrm{Na}]^{+}, 38\right)$. Mass required for $\mathrm{C}_{22} \mathrm{H}_{29} \mathrm{FeN}_{4} \mathrm{O}: 421$, 
found: 421. Anal. Calcd. for $\mathrm{C}_{22} \mathrm{H}_{28} \mathrm{FeN}_{4} \mathrm{O}$ : C, 62.86; H, 6.71; N, 13.33. Found: C, 63.07; H, 6.52; N, 13.29 .

1-Cyclohexyl-3-(4-ferrocenyl-6-propylpyrimidin-2-yl)-urea (5k). Yield: $80 \%$. Red solid. Mp: $55-58{ }^{\circ} \mathrm{C}$. $\delta_{\mathrm{H}}\left(400 \mathrm{MHz} \mathrm{CDCl}_{3}\right): 9.34$ (d, J=7.5 Hz, $1 \mathrm{H}, \mathrm{NH}), 7.21(\mathrm{~s}, 1 \mathrm{H}, \mathrm{NH}), 6.73(\mathrm{~s}, 1 \mathrm{H}, 5-\mathrm{H}), 4.87(\mathrm{t}$, $J=1.8 \mathrm{~Hz}, 2 \mathrm{H}, \mathrm{Cp}), 4.50(\mathrm{t}, J=1.8 \mathrm{~Hz}, 2 \mathrm{H}, \mathrm{Cp}), 4.07\left(\mathrm{~s},{ }_{5} \mathrm{H}, \mathrm{Cp}\right)$, 3.78-3.87 (m, $1 \mathrm{H}, \mathrm{cHex}), 2.61\left(t, \mathrm{~J}=7.4 \mathrm{~Hz}, 2 \mathrm{H}, \mathrm{C} 6-\mathrm{CH}_{2}\right), 2.03-2.14$ (m, $2 \mathrm{H}, \mathrm{cHex}), 1.73-1.84\left(\mathrm{~m}, 4 \mathrm{H}, \mathrm{cHex},-\mathrm{CH}_{2}-\mathrm{CH}_{3}\right), 1.62-1.70(\mathrm{~m}$, $1 \mathrm{H}, \mathrm{cHex}), 1.25-1.52(\mathrm{~m}, 5 \mathrm{H}, \mathrm{cHex}), 0.99\left(\mathrm{t}, \mathrm{J}=7.4 \mathrm{~Hz},{ }_{3} \mathrm{H}, \mathrm{CH}_{3}\right)$. $\delta_{\mathrm{C}}\left(100 \mathrm{MHz}, \mathrm{CDCl}_{3}\right): 170.3,168.2,157.7,153.9,106.9,79.8,71.1$, 70.0, 67.9, 48.6, 39.5, 33.6, 25.7, 24.8, 21.7, 13.7. $v_{\max }\left(\mathrm{KBr} ; \mathrm{cm}^{-1}\right)$ : 3438, 3223, 3081, 1682, 1590, 1531. ESI MS ( $\mathrm{m} / \mathrm{z}$ (relative intensity)) $447\left([\mathrm{M}+\mathrm{H}]^{+}, 30\right), 469\left([\mathrm{M}+\mathrm{Na}]^{+}, 100\right), 915\left([2 \mathrm{M}+\mathrm{Na}]^{+}, 18\right)$. Mass required for $\mathrm{C}_{24} \mathrm{H}_{31} \mathrm{FeN}_{4} \mathrm{O}$ : 447, found: 447. Anal. Calcd. for $\mathrm{C}_{24} \mathrm{H}_{30} \mathrm{FeN}_{4} \mathrm{O}$ : C, 64.58; H, 6.77; N, 12.55. Found: C, 64.55; H, 6.47; $\mathrm{N}, 12.71$.

1-(3-Chloropropyl)-3-(4-ferrocenyl-6-propylpyrimidin-2-yl)-urea (5l). Yield: $99 \%$. Red oil. $\delta_{\mathrm{H}}\left(400 \mathrm{MHz}, \mathrm{CDCl}_{3}\right): 9.53(\mathrm{t}, J=5.5 \mathrm{~Hz}$, $1 \mathrm{H}, \mathrm{NH}), 7.25(\mathrm{~s}, 1 \mathrm{H}, \mathrm{NH}), 6.76(\mathrm{~s}, 1 \mathrm{H}, 5-\mathrm{H}), 4.88(\mathrm{t}, J=1.9 \mathrm{~Hz}, 2 \mathrm{H}$, $\mathrm{Cp}), 4.50(\mathrm{t}, \mathrm{J}=1.9 \mathrm{~Hz}, 2 \mathrm{H}, \mathrm{Cp}), 4.08\left(\mathrm{~s},{ }_{5} \mathrm{H}, \mathrm{Cp}\right), 3.71(\mathrm{t}, J=6.3 \mathrm{~Hz}$, $2 \mathrm{H}, \mathrm{Cl}-\mathrm{CH}_{2}$ ), 3.60 (qua, $\left.J=6.3 \mathrm{~Hz}, 2 \mathrm{H}, \mathrm{NH}-\mathrm{CH}_{2}\right), 2.63(\mathrm{t}, J=7.4 \mathrm{~Hz}$, $2 \mathrm{H}, \mathrm{C} 6-\mathrm{CH}_{2}$ ), 2.13 (qui, J=6.3 Hz, $2 \mathrm{H}, \mathrm{CH}_{2}-\mathrm{CH}_{2}-\mathrm{Cl}$ ), 1.76 (sext, $\left.J=7.4 \mathrm{~Hz}, 2 \mathrm{H}, \mathrm{CH}_{2}-\mathrm{CH}_{3}\right)$, $1.01\left(\mathrm{t}, J=7.4 \mathrm{~Hz},{ }_{3} \mathrm{H}, \mathrm{CH}_{3}\right) . \delta_{\mathrm{C}}(100 \mathrm{MHz}$, $\left.\mathrm{CDCl}_{3}\right): 170.3,168,5,157.5,155.3,109.2,79.6,71.2,70.0,68.0,42.6$, 39.5, 37.0, 32.4, 21.7, 13.7. $v_{\max }\left(\mathrm{KBr} ; \mathrm{cm}^{-1}\right): 3436,3222,3089,1685$, 1591, 1534. ESI MS $\left(m / z\right.$ (relative intensity)) $441\left([\mathrm{M}+\mathrm{H}]^{+}, 42\right), 477$ $\left([\mathrm{M}+\mathrm{Na}]^{+}, \quad 100\right), 903\left([2 \mathrm{M}+\mathrm{Na}]^{+}, \quad 50\right)$. Mass required for $\mathrm{C}_{21} \mathrm{H}_{26} \mathrm{ClFeN}_{4} \mathrm{O}: 441$, found 441. Anal. Calcd. for $\mathrm{C}_{21} \mathrm{H}_{25} \mathrm{ClFeN}_{4} \mathrm{O}$ : C, 57.23 H, 5.72; N, 12.71. Found: C, 57.12; H, 5.90; N, 12.84 .

1-(4-tert-Butyl-6-ferrocenylpyrimidin-2-yl)-3-phenylurea (5m). Yield: $94 \%$. Dark red solid. Mp: $86-89{ }^{\circ} \mathrm{C}$. $\delta_{\mathrm{H}}\left(400 \mathrm{MHz}, \mathrm{CDCl}_{3}\right)$ : 11.64 (s, $1 \mathrm{H}, \mathrm{NH}), 7.67$ (d, J=7.4 Hz, $2 \mathrm{H}, \mathrm{Ph}), 7.35-7.40\left(\mathrm{~m},{ }_{3} \mathrm{H}, \mathrm{Ph}\right.$, NH), $7.11(\mathrm{tt}, J=7.4 \mathrm{~Hz}, J=1.1 \mathrm{~Hz}, 1 \mathrm{H}, \mathrm{Ph}), 6.95(\mathrm{~s}, 1 \mathrm{H}, 5-\mathrm{H}), 4.95(\mathrm{t}$, $J=1.9 \mathrm{~Hz}, 2 \mathrm{H}, \mathrm{Cp}), 4.53(\mathrm{t}, J=1,9 \mathrm{~Hz}, 2 \mathrm{H}, \mathrm{Cp}), 4.09$ (s, $5 \mathrm{H}, \mathrm{Cp}), 1.41$ $\left(\mathrm{s}, 9 \mathrm{H}, \mathrm{C}\left(\mathrm{CH}_{3}\right)_{3}\right) . \delta_{\mathrm{C}}\left(100 \mathrm{MHz}, \mathrm{CDCl}_{3}\right): 177.0,169.9,157.0,152.3$, 138.3, 129.0, 123.4, 119.9, 106.0, 80.6, 72.0, 70.8, 68.7, 37.4, 29.5. $v_{\max }\left(\mathrm{KBr} ; \mathrm{cm}^{-1}\right): 3223,3179,3138,3107,1685,1590,1534$. ESI MS $(\mathrm{m} / \mathrm{z}$ (relative intensity) $) 455\left([\mathrm{M}+\mathrm{H}]^{+}, 100\right), 477\left([\mathrm{M}+\mathrm{Na}]^{+}, 75\right)$, $931\left([2 \mathrm{M}+\mathrm{Na}]^{+}, 20\right)$. Mass required for $\mathrm{C}_{25} \mathrm{H}_{27} \mathrm{FeN}_{4} \mathrm{O}: 455$, found: 455. Anal. Calcd. for $\mathrm{C}_{25} \mathrm{H}_{26} \mathrm{FeN}_{4} \mathrm{O}: \mathrm{C}, 66.09 ; \mathrm{H}, 5.77 ; \mathrm{N}, 12.33$. Found: C, 66.15; H, 5.62; N, 12.35 .

1-Butyl-3-(4-tert-butyl-6-ferrocenylpyrimidin-2-yl)-urea (5n). Yield: $90 \%$. Red solid. Mp: $46-48{ }^{\circ} \mathrm{C}$. $\delta_{\mathrm{H}}\left(400 \mathrm{MHz}, \mathrm{CDCl}_{3}\right): 9.32$ (t, J=5.5 Hz, 1H, NH), $7.26(\mathrm{~s}, 1 \mathrm{H}, \mathrm{NH}), 6.88(\mathrm{~s}, 1 \mathrm{H}, 5-\mathrm{H}), 4.88(\mathrm{t}$, $J=1.8 \mathrm{~Hz}, 2 \mathrm{H}, \mathrm{Cp}), 4.49(\mathrm{t}, J=1.8 \mathrm{~Hz}, 2 \mathrm{H}, \mathrm{Cp}), 4.67$ (s, $5 \mathrm{H}, \mathrm{Cp}), 3.42$ (qua, $J=7.3 \mathrm{~Hz}, 2 \mathrm{H}, \mathrm{NH}-\underline{\mathrm{CH}}_{2}$ ), 1.65 (qui, $J=7.3 \mathrm{~Hz}, 2 \mathrm{H}, \mathrm{NH}-$ $\mathrm{CH}_{2} \mathrm{CH}_{2}$ ), 1.49 (sext, J=7.3 Hz, $2 \mathrm{H}, \mathrm{NH}-\mathrm{CH}_{2} \mathrm{CH}_{2} \underline{\mathrm{CH}}_{2}$ ), 1.34 (s, $9 \mathrm{H}$, $\left.\mathrm{C}\left(\mathrm{CH}_{3}\right)_{3}\right), 0.98\left(\mathrm{t}, J=7.3 \mathrm{~Hz}, 3 \mathrm{H}, \mathrm{CH}_{3}\right) . \delta_{\mathrm{C}}\left(100 \mathrm{MHz}, \mathrm{CDCl}_{3}\right): 177.2$, 168.9, 157.3, 155.1, 105.4, 80.2, 71.0, 70.0, 67.9, 39.7, 37.2, 32.0, 29.3, 20.1, 13.7. $v_{\max }\left(\mathrm{KBr} ; \mathrm{cm}^{-1}\right): 3438,3234,3100,1685,1586,1528$. ESI MS $\left(\mathrm{m} / \mathrm{z}\right.$ (relative intensity)) $435\left([\mathrm{M}+\mathrm{H}]^{+}, 38\right), 457\left([\mathrm{M}+\mathrm{Na}]^{+}\right.$, 100), $891\left([2 \mathrm{M}+\mathrm{Na}]^{+}, 18\right)$. .Mass required for $\mathrm{C}_{23} \mathrm{H}_{31} \mathrm{FeN}_{4} \mathrm{O}: 435$, found: 435. Anal. Calcd. for $\mathrm{C}_{23} \mathrm{H}_{30} \mathrm{FeN}_{4} \mathrm{O}: \mathrm{C}, 63.60 ; \mathrm{H}, 6.96 ; \mathrm{N}$, 12.90. Found: C, 63.46; H, 7.11; N, 12.95 .

1-(4-tert-Butyl-6-ferrocenylpyrimidin-2-yl)-3-cyclohexylurea (5o). Yield: $92 \%$. Red solid. Mp: $118-120{ }^{\circ} \mathrm{C} . \delta_{\mathrm{H}}\left(400 \mathrm{MHz}, \mathrm{CDCl}_{3}\right)$ : $9.29(\mathrm{~d}, J=7.7 \mathrm{~Hz}, \mathrm{iH}, \mathrm{NH}), 7.18(\mathrm{~s}, 1 \mathrm{H}, \mathrm{NH}), 6.87$ (s, $1 \mathrm{H}, 5-\mathrm{H}), 4.89$ (t, J=1.9 Hz, 2H, Cp), 4.49 (t, J=1.9 Hz, 2H, Cp), 4.07 (s, 5H, Cp), 3.74-3.84 (m, $1 \mathrm{H}$, cHex), 2.07-2.16 (m, 2H, cHex), 1.78-1.86 (m, $2 \mathrm{H}, \mathrm{cHex}$ ), 1.65-1.72 (m, 1H, cHex), 1.37-1.50 (m, 2H, cHex), 1.34 (s, $\left.{ }_{9} \mathrm{H}, \mathrm{C}\left(\mathrm{CH}_{3}\right)_{3}\right), 1.18-1.32(\mathrm{~m}, 3 \mathrm{H}, \mathrm{cHex}) . \delta_{\mathrm{C}}\left(100 \mathrm{MHz}, \mathrm{CDCl}_{3}\right): 177.1$, 169.0, 157.3, 154.1, 105.3, 80.2, 71.0, 69.9, 67.9, 48.9, 37.2, 33.9, 29.3, 25.6, 25.1. $v_{\max }\left(\mathrm{KBr} ; \mathrm{cm}^{-1}\right): 3472,3221,3120,1675,1586,1525$. ESI MS $\left(m / z\right.$ (relative intensity)) $461\left([\mathrm{M}+\mathrm{H}]^{+}, 56\right), 483\left([\mathrm{M}+\mathrm{Na}]^{+}\right.$, 100), $943\left([2 \mathrm{M}+\mathrm{Na}]^{+}, 66\right)$. Mass required for $\mathrm{C}_{25} \mathrm{H}_{33} \mathrm{FeN}_{4} \mathrm{O}: 461$, found: 461. Anal. Calcd. for $\mathrm{C}_{25} \mathrm{H}_{32} \mathrm{FeN}_{4} \mathrm{O}: \mathrm{C}, 65.22 ; \mathrm{H}, 7.01 ; \mathrm{N}$, 12.17. Found: C, 64.95; H, 7.06; N, 12.31.
1-(4-tert-Butyl-6-ferrocenylpyrimidin-2-yl)-3-(3-

chloropropyl)urea (5p). Yield: $95 \%$. Red solid. Mp: $34-36{ }^{\circ} \mathrm{C} . \delta_{\mathrm{H}}$ $\left(400 \mathrm{MHz}, \mathrm{CDCl}_{3}\right): 9.53(\mathrm{t}, J=5.6 \mathrm{~Hz}, 1 \mathrm{H}, \mathrm{NH}), 7.25(\mathrm{~s}, 1 \mathrm{H}, \mathrm{NH})$, $6.90(\mathrm{~s}, 1 \mathrm{H}, 5-\mathrm{H}), 4.90(\mathrm{t}, J=1.9 \mathrm{~Hz}, 2 \mathrm{H}, \mathrm{Cp}), 4.49(\mathrm{t}, \mathrm{J}=1.9 \mathrm{~Hz}, 2 \mathrm{H}$, $\mathrm{Cp}$ ), $4.07(\mathrm{~s}, 5 \mathrm{H}, \mathrm{Cp}), 3.69\left(\mathrm{t}, J=6.4 \mathrm{~Hz}, 2 \mathrm{H}, \mathrm{Cl}-\mathrm{CH}_{2}\right.$ ), 3.59 (qua, $J=6.4 \mathrm{~Hz}, 2 \mathrm{H}, \mathrm{NH}-\mathrm{C}_{2}$ ), 2.13 (qui, J=6.4 Hz, $2 \mathrm{H}, \mathrm{CH}_{2}-\mathrm{CH}_{2}-\mathrm{Cl}$ ), 1.35 $\left(\mathrm{s}, 9 \mathrm{H}, \mathrm{C}\left(\mathrm{CH}_{3}\right)_{3}\right) . \delta_{\mathrm{C}}\left(100 \mathrm{MHz}, \mathrm{CDCl}_{3}\right): 177.7,169.2,157 \cdot 3,154.9$, 105.6, 80.2, 71.2, 70.1, 68.0, 42.4, 37.4, 37.0, 32.5, 29.4. $v_{\max }(\mathrm{KBr}$; $\left.\mathrm{cm}^{-1}\right): 3436,3219,3113,089,1684,1587,1520$. ESI MS ( $\mathrm{m} / \mathrm{z}$ (relative intensity $) 455\left([\mathrm{M}+\mathrm{H}]^{+}, 60\right), 477\left([\mathrm{M}+\mathrm{Na}]^{+}, 100\right), 931\left([2 \mathrm{M}+\mathrm{Na}]^{+}\right.$, 23).(Mass required for $\mathrm{C}_{22} \mathrm{H}_{28} \mathrm{ClFeN}_{4} \mathrm{O}$ : 455, found: 455. Anal. Calcd. for $\mathrm{C}_{22} \mathrm{H}_{27} \mathrm{ClFeN}_{4} \mathrm{O}$ : $\mathrm{C}, 58.10 ; \mathrm{H}, 5.98 ; \mathrm{N}, 12.32$. Found: $\mathrm{C}$, 57.96; H, 6.14; N, 12.21.

X-ray Structural Analysis. Suitable crystals of $\mathbf{5 m}$ were grown from toluene. At room temperature, crystals degraded rapidly before the end of data collection so that data were collected at low temperature $(15 \mathrm{OK})$ on a Gemini diffractometer (Oxford Diffraction Ltd) equipped with a Ruby CCD detector using Enhance Mo X-ray Source. Structure has been refined on $F^{2}$ using the SHELXL-2014 ${ }^{22}$ suite of programs and data analysis was performed with PLATON. ${ }^{23}$ The compound crystallized as a solvate. The toluene solvent molecule is highly disordered in the structure. A multi-scan procedure was applied to correct for absorption effects. Hydrogen atom positions were calculated and refined isotropically using a riding model. CCDC-1483397 entry contains the supplementary crystallographic data for this paper. These data can be obtained free of charge from The Cambridge Crystallographic Data Centre via www.ccdc.cam.ac.uk/data_request/cif.

Electrochemical Measurements. Electrochemical measurements were performed on a Radio meter Analytical PST-oo6 instrument (software: Volamaster 4), with a conventional threeelectrode configuration consisting of a spectral graphite $(\mathrm{OD}=$ $3 \mathrm{~mm}$ ) working electrode, a platinum auxiliary electrode and a $\mathrm{Ag} / \mathrm{AgCl}$ reference electrode. The experiments were carried out in $1 \mathrm{mM}$ solution of the samples in $\mathrm{CH}_{2} \mathrm{Cl}_{2}, \mathrm{CH}_{3} \mathrm{CN}$ or in mixtures thereof, containing o.1 $\mathrm{M}\left(\mathrm{n}-\mathrm{C}_{4} \mathrm{H}_{9}\right)_{4} \mathrm{NClO}_{4}\left(\mathrm{TBAClO}_{4}\right)$ as supporting electrolyte (cell volume: 1oml). Titration experiments were carried out using $\mathrm{CH}_{2} \mathrm{Cl}_{2}$ solutions of the host $5 \mathrm{~g}(0.5 \mathrm{mM})$ and guest 6 (concentrations between $0.125 \mathrm{mM}$ and $5 \mathrm{mM}$ ). For deoxygenation, the solutions were sonicated under argon and kept in closed vessels under argon atmosphere as well. The working electrode was wet polished on $0.5 \mu \mathrm{m}$ alumina slurry, or sand paper respectively after each run. CV and LSV measurements were carried out with a scan rate of $0.10 \mathrm{~V} \mathrm{~s}^{-1}$. CAUTION: $\mathrm{TBAClO}_{4}$ may cause skin-, eye- and respiratory irritation and may intensify fire. (Keep away from combustible materials. Avoid breathing dust. Wear eyeshields, protective gloves and full-face particle respirator.)

Computational Methods. Theoretical calculations were performed with the Gaussian og software package. ${ }^{24}$ Molecular geometries were optimized using the long-range corrected hybrid density functional CAM-B $3 L_{Y} P^{25}$ and the standard $6-31 G^{*}$ basis set. ${ }^{26}$ The optimizations were performed without any symmetry constraint. The vibrational analysis yielded no imaginary frequencies verifying that each of the optimised structures is a true minimum on the potential energy surface. Gibbs free energies have been computed at $298 \mathrm{~K}$. For the graphical visualization, the molden program was used. ${ }^{27}$ The choice of the method was tested by optimizing the geometry of compound $5 \mathrm{~m}$ starting from the XRD structure.

\section{RESULTS AND DISCUSSION}

Synthesis. New 2-ureido-4-ferrocenyl-pyrimidine derivatives were prepared in the present study using the twostep synthetic method developed earier in our groupfor 
preparation of ferrocenyl-pyrimidine compounds. ${ }^{18 a}$ Moreover, some features of the previous methodology, leading to the intermediate 2-amino-pyrimidines, have been improved.

Iodoferrocene (1, Scheme 1) was converted into ferrocenyl-alkynyl ketones (3a-d) by a carbonylative Sonogashira coupling. The products were obtained in 65$91 \%$ yield depending on the alkyne reaction partner (Table 1). Besides the usual homogeneous catalytic conditions, the coupling of $\mathbf{1}$ with $\mathbf{2 a}$ was carried out in the presence of a supported catalyst (SILP-Pd, Fig 1.), prepared by the immobilization of palladium on ionic liquid modified silica. ${ }^{20} \mathrm{With}$ the use of this heterogeneous catalyst, 3a could be obtained in $83 \%, 86 \%$ and $76 \%$ yields in three subsequent runs with the recycling of the catalyst under copper free conditions. (Table 1, entries 2-4). Although these yields are a bit lower than those obtained by the homogeneous system, the TON is much higher (a total of 153 for the three cycles) because of the lower palladium content of the catalyst (1.6\%) compared to the homogeneous system (10\%).

2-Amino-4-ferrocenyl pyrimidines (4a-d) were obtained by the reaction of alkynyl ketones $3 \mathbf{a}-\mathbf{d}$ with guanidine carbonate in refluxing ethanol (Scheme 1, Table 2). The application of guanidine carbonate instead of the hydrochloride salt ${ }^{18 a}$ eliminated the need for an added base and resulted in better yields of the known compounds $4 \mathbf{a}$ and 4b than before.

2-Amino-pyrimidines (4a-d) were converted into the products $\mathbf{5} \mathbf{a}-\mathbf{p}$ by simply heating them in an access of the corresponding isocyanate (Scheme 1, Table 3 ). It should be mentioned that the usual conditions ( 7 eq isocyanate in pyridine; $\left.100^{\circ} \mathrm{C} ; 21 \mathrm{~h}\right)^{28}$ led to the products in much lower yield (e.g. 8\% in case of 5 a instead of the $77 \%$ obtained under solvent-free conditions).
Scheme 1. Synthesis of 6-substituted 2-ureido-4ferrocenyl pyrimidines $5 \mathbf{a}-\mathbf{p}$
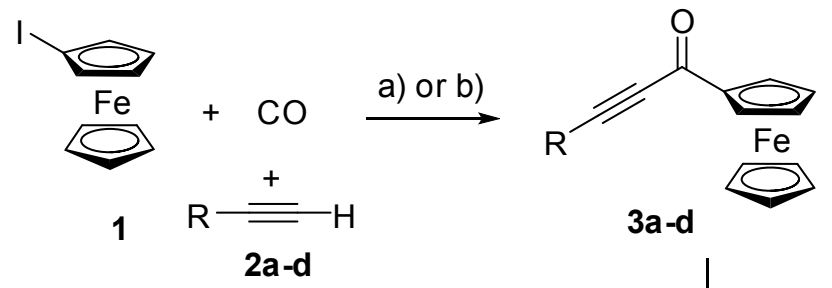

c)

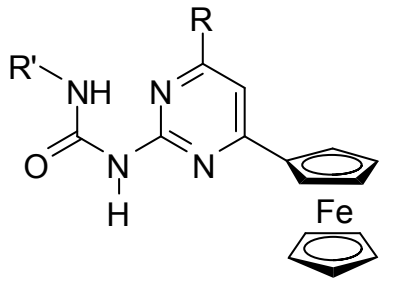

$5 a-p$

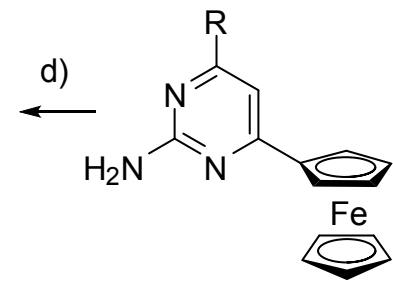

4a-d
Reaction conditions: a) $10 \% \mathrm{PdCl}_{2}\left(\mathrm{PPh}_{3}\right)_{2}, 4 \% \mathrm{CuI}$, THF, $\mathrm{Et}_{3} \mathrm{~N}, 15$ bar, $60{ }^{\circ} \mathrm{C}, 12 \mathrm{~h}$; b) SILP-Pd (1.6\% Pd) THF, Et ${ }_{3} \mathrm{~N}, 15$ bar, $\left.60{ }^{\circ} \mathrm{C}, 12 \mathrm{~h}, \mathrm{c}\right)$ guanidine carbonate, EtOH, reflux, $12 \mathrm{~h}$; d) R'NCO, $100{ }^{\circ} \mathrm{C}, 12 \mathrm{~h}$.

Table 1. Synthesis of ferrocenyl-alkynyl-ketones via carbonylative Sonogashira coupling ${ }^{\mathrm{a}}$

\begin{tabular}{|l|l|l|l|l|}
\hline entry & $\mathrm{R}$ & catalyst & product & yield (\%) \\
\hline $\mathbf{1}$ & $\mathrm{Ph}$ & $\mathrm{A}$ & $\mathbf{3 a}$ & 91 \\
\hline $\mathbf{2}$ & $\mathrm{Ph}$ & $\mathrm{B}$ & $\mathbf{3 a}$ & 83 \\
\hline 3 & $\mathrm{Ph}$ & $\mathrm{B}^{\mathrm{b}}$ & $\mathbf{3 a}$ & 86 \\
\hline 4 & $\mathrm{Ph}$ & $\mathrm{B}^{\mathrm{c}}$ & $\mathbf{3 a}$ & 76 \\
\hline 5 & $\mathrm{nBu}$ & $\mathrm{A}$ & $\mathbf{3 b}$ & 65 \\
\hline 6 & $\mathrm{nPr}$ & $\mathrm{A}$ & $\mathbf{3 c}$ & 78 \\
\hline 7 & $\mathrm{tBu}$ & $\mathrm{A}$ & $\mathbf{3 d}$ & 85 \\
\hline
\end{tabular}

a: reaction conditions: catalyst $\left(\mathrm{A}: 10 \% \mathrm{PdCl}_{2}\left(\mathrm{PPh}_{3}\right)_{2}, 4 \%\right.$ CuI; B: SILP-Pd (1.6\% Pd)) THF, Et ${ }_{3} \mathrm{~N}, 15$ bar, $60{ }^{\circ} \mathrm{C}, 12 \mathrm{~h} ;{ }^{\mathrm{b}}: 1^{\text {st }}$ recycle; ${ }^{\text {c }} 2^{\text {nd }}$ recycle

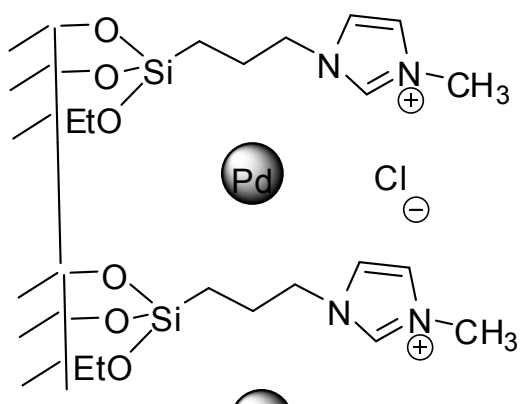

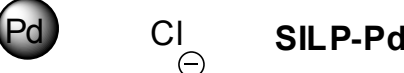


Figure 1. The structure of the SILP-Pd catalyst used in the carbonylative Sonogashira reaction

Table 2. Synthesis of 2-amino-4ferrocenylpyrimidines ${ }^{\text {a }}$

\begin{tabular}{|l|l|l|l|}
\hline entry & $\mathrm{R}$ & product & yield (\%) \\
\hline $\mathbf{1}$ & $\mathrm{Ph}$ & $\mathbf{4 a}$ & 54 \\
\hline $\mathbf{2}$ & $\mathrm{nBu}$ & $\mathbf{4 b}$ & 85 \\
\hline 3 & $\mathrm{nPr}$ & $\mathbf{4 c}$ & 78 \\
\hline 4 & $\mathrm{tBu}$ & $\mathbf{4 d}$ & 67 \\
\hline
\end{tabular}

a: reaction conditions: guanidine carbonate (2.2 eq), EtOH, reflux, $12 \mathrm{~h}$.

Table 3. Synthesis of 2-ureido-4ferrocenylpyrimidines ${ }^{\mathrm{a}}$

\begin{tabular}{|c|c|c|c|c|c|}
\hline entry & $\mathrm{R}$ & $\mathrm{R}^{\prime}$ & $\begin{array}{l}\text { reaction } \\
\text { time }\end{array}$ & product & $\begin{array}{l}\text { yield } \\
(\%)\end{array}$ \\
\hline 1 & $\mathrm{Ph}$ & $\mathrm{Ph}$ & $10 \mathrm{~min}$ & $5 a$ & 77 \\
\hline 2 & $\mathrm{Ph}$ & $\mathrm{nBu}$ & $6 \mathrm{~h}$ & $5^{b}$ & 68 \\
\hline 3 & $\mathrm{Ph}$ & cHex & $3 \mathrm{~h}$ & $5 \mathrm{C}$ & 67 \\
\hline 4 & $\mathrm{Ph}$ & $\left(\mathrm{CH}_{2}\right)_{3} \mathrm{Cl}$ & $30 \mathrm{~min}$ & $5 d$ & 97 \\
\hline 5 & $\mathrm{nBu}$ & $\mathrm{Ph}$ & $10 \mathrm{~min}$ & $5 e$ & 99 \\
\hline 6 & $\mathrm{nBu}$ & $\mathrm{nBu}$ & $3 \mathrm{~h}$ & $5 f$ & 83 \\
\hline 7 & $\mathrm{nBu}$ & cHex & $2 \mathrm{~h}$ & $5 g$ & 84 \\
\hline 8 & $\mathrm{nBu}$ & $\left(\mathrm{CH}_{2}\right)_{3} \mathrm{Cl}$ & $10 \mathrm{~min}$ & $5 \mathrm{~h}$ & 94 \\
\hline 9 & $\mathrm{nPr}$ & $\mathrm{Ph}$ & $10 \mathrm{~min}$ & $5^{\mathbf{i}}$ & 99 \\
\hline 10 & $\mathrm{nPr}$ & $\mathrm{nBu}$ & $3 \mathrm{~h}$ & $5 \mathbf{j}$ & 82 \\
\hline 11 & $\mathrm{nPr}$ & cHex & $2 \mathrm{~h}$ & $5^{k}$ & 80 \\
\hline 12 & $\mathrm{nPr}$ & $\left(\mathrm{CH}_{2}\right)_{3} \mathrm{Cl}$ & $10 \mathrm{~min}$ & $5^{1}$ & 99 \\
\hline 13 & $\mathrm{tBu}$ & $\mathrm{Ph}$ & $10 \mathrm{~min}$ & $5 \mathrm{~m}$ & 94 \\
\hline 14 & $\mathrm{tBu}$ & $\mathrm{nBu}$ & $2 \mathrm{~h}$ & $5^{n}$ & 90 \\
\hline 15 & $\mathrm{tBu}$ & cHex & $1 \mathrm{~h}$ & 50 & 92 \\
\hline 16 & $\mathrm{tBu}$ & $\left(\mathrm{CH}_{2}\right)_{3} \mathrm{Cl}$ & $10 \mathrm{~min}$ & $5 P$ & 95 \\
\hline
\end{tabular}

${ }^{\mathrm{a}}$ : reaction conditions: $\mathrm{R}^{\prime} \mathrm{NCO}(7 \mathrm{eq}),. 100^{\circ} \mathrm{C}$

Characterization. The structures of compounds 3 a-d, 4a-d and 5a-p have been confirmed by the usual techniques ( ${ }^{1} \mathrm{H}$ NMR, ${ }^{13} \mathrm{C}$ NMR, IR, MS, and elemental analysis).

The ${ }^{1} \mathrm{H}$ NMR spectra of pyrimidines 5 in $\mathrm{CDCl}_{3}$ show two $\mathrm{NH}$ signals, one in the region of 9.29-11.75 ppm and another one in the region of 7.19-7.53 ppm corresponding to $\mathrm{H}$ bonded and non-H-bonded NH protons, respectively. The signal with the high downfield shift corresponds to the R'NH proton of compounds 5, exhibiting a singlet, doublet or triplet pattern depending on the structure of the R' substituent. The change of the chemical shift of this proton is within 0.01 ppm on 6-fold dilution with $\mathrm{CDCl}_{3}$ that supports the presence of an intramolecular interaction (for the spectra of $\mathbf{5 g}$ see supporting information, Figure $\mathrm{S}_{1}$ ). On heating, a -4.1 ppb $\mathrm{K}^{-1} \Delta \delta \mathrm{H}$ (temperature range: $303-353 \mathrm{~K}$ ) was observed in

toluene- $\mathrm{d}_{8}$ that shows the existence of a strong hydrogen bond (Figure $\mathrm{S}_{2}$ ).

Crystals of compound $\mathbf{5 m}$ suitable for X-ray analysis were grown in toluene. Figure 2 shows its molecular structure and main crystallographic data are provided in Table 4 . The compound crystallized as a solvate. In the solid state, the existence of an intramolecular hydrogen bond was confirmed by $\mathrm{X}$-ray diffraction (XRD) analysis.

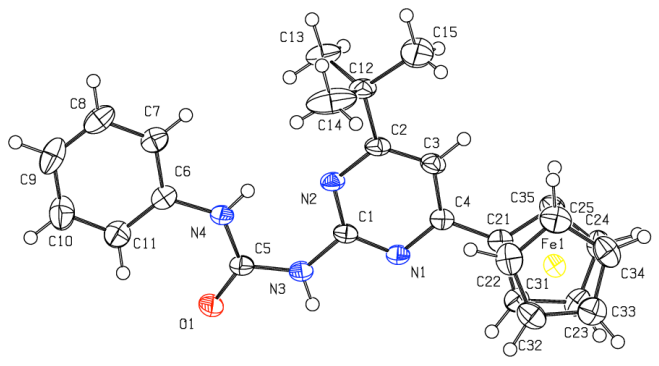

Figure 2. X-ray structure (ORTEP diagram drawn at 50\% probability) of compound $\mathbf{5 m}$. The solvent (toluene) molecule is not presented for clarity.

The $C p$ rings are arranged in an eclipsed conformation (the $\mathrm{C}_{21}$-centroid $\mathrm{C}_{\mathrm{p}}(21-25)$-centroid $\mathrm{C}_{\mathrm{p}}(31-35)-\mathrm{C}_{31}$ torsion angle is $6.7^{\circ}$ ) and are almost parallel (the tilt angle is $2.0^{\circ}$ ). The pyrimidine ring is out of the plane of the $\mathrm{Cp}$ ring it is connected to, with an $\mathrm{N}_{1}-\mathrm{C}_{4}-\mathrm{C}_{21}-\mathrm{C}_{22}$ torsion angle of 17.0 $(4)^{\circ}$. The pyrimidine ring, the ureido group and the phenyl ring are almost coplanar and only slightly tilted by angles of $3.0^{\circ}$ and $8.1^{\circ}$, respectively (torsion angle $\mathrm{C}_{1}-\mathrm{N}_{3}-\mathrm{C}_{5}-\mathrm{O}_{1}$ : $177.9(3)^{\circ}$, torsion angle $\left.\mathrm{C}_{1}-\mathrm{N}_{3}-\mathrm{C}_{5}-\mathrm{N}_{4}:-2.3(4)^{\circ}\right)$. An intramolecular hydrogen bond is formed between the $\mathrm{N}_{2}$ nitrogen atom of the pyrimidine ring and the $\mathrm{N}_{4} \mathrm{NH}$ donor group of the urea moiety ( $\mathrm{N}_{2}-\mathrm{N}_{4}$ distance: $2.690(3) \AA$; $\mathrm{N}_{2}-\mathrm{H}_{4}$ distance: $1.980 \AA$ A; $\mathrm{N}_{2}-\mathrm{H}_{4}-\mathrm{N}_{4}$ angle: $\left.139.00^{\circ}\right)$.

Two monomers form a dimer by intermolecular hydrogen bonds between the carbonyl oxygen atom of one monomer and an $\mathrm{NH}$ donor group of the other $\left(\mathrm{N}_{3}{ }^{\prime}-\mathrm{O}_{1}\right.$ distance

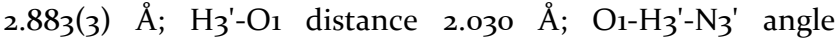
$\left.173.00^{\circ}\right)$. The unsubstituted $\mathrm{Cp}$ rings of the ferrocene units are located on the opposite sides of the plane of the H-bonds.

Table 4. Summary of crystallographic data for $5 \mathrm{~m}$

Formula

Formula Weight

Crystal System

Space group

$\mathrm{a}, \mathrm{b}, \mathrm{c}[\AA]$

$\alpha, \beta, \gamma\left[{ }^{\circ}\right]$

$\mathrm{V}[$ Ang $* * 3]$

$\mathrm{Z}$

$\mathrm{D}$ (calc) $\left[\mathrm{g} / \mathrm{cm}^{3}\right]$

$\mathrm{Mu}\left(\mathrm{MoK}_{a}\right)[/ \mathrm{mm}]$

$\mathrm{F}(000)$

Crystal Size [mm]

\section{Crystal Data}

$$
\begin{array}{cc}
\mathrm{C}_{25} \mathrm{H}_{26} \mathrm{Fe} \mathrm{N}_{4} \mathrm{O}, \mathrm{C}_{7} \mathrm{H}_{8} \\
546.48 \\
\multicolumn{3}{c}{\text { Triclinic }} \\
\mathrm{P}-1 \quad \text { (No. 2) }
\end{array}
$$

9.3228(4) $11.0259(6) \quad 14.7499(7)$

$90.484(4) \quad 103.348(4) \quad 107.081(4)$

$1405.40(13)$

2

1.291

0.568

576

$0.08 \times 0.11 \times 0.54$ 


\begin{tabular}{|c|c|}
\hline \multicolumn{2}{|l|}{ Data Collection } \\
\hline Temperature $(\mathrm{K})$ & 150 \\
\hline Radiation $[\AA]$ & 0.71073 \\
\hline Tot., Uniq. Data, R(int) & $9699, \quad 4950,0.030$ \\
\hline Observed data $[\mathrm{I}>2.0 \operatorname{sigma}(\mathrm{I})]$ & 4339 \\
\hline \multicolumn{2}{|l|}{ Refinement } \\
\hline Nref, Npar & 4950,347 \\
\hline $\mathrm{R}, \mathrm{wR} 2, \mathrm{~S}$ & $0.0478,0.1233,1.03$ \\
\hline Max. and Av. Shift/Error & $0.00,0.00$ \\
\hline Min. and Max. Resd. Dens. [e/Å3 & $-0.45,0.97$ \\
\hline
\end{tabular}

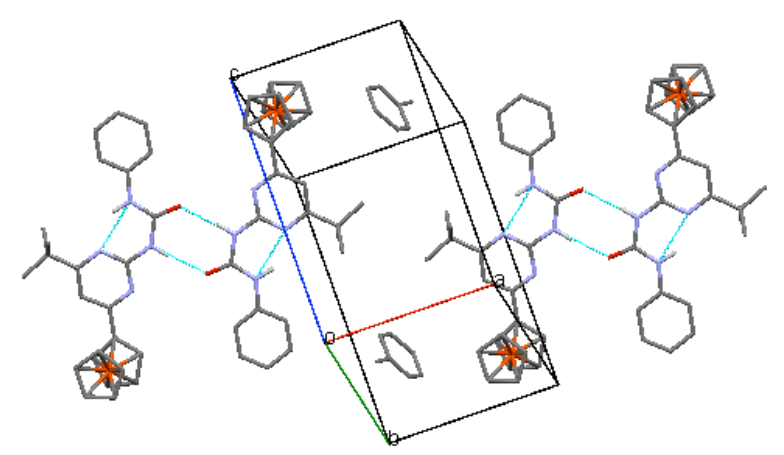

Figure 3. Crystal packing of compound $\mathbf{5 m}$ illustrating a layer of compounds formed by dimeric $\mathrm{H}$-bonded units. H-bonds are represented by blue dashed lines.

The crystal packing of $\mathbf{5 m}$ (Figures 3 and $\mathrm{S}_{3}$ ) consists of pairs of close-to-planar dimers described above, arranged in a stair-like structure along the c-axis in which two slipped dimers with parallel cyclopentadienyl ring-pyrimidine ringureido group-phenyl ring planes form a unit. They are connected in a 'head to tail' manner, with twofold stabilizing weak interactions between adjacent layers. The first weak $\mathrm{CH}$... $\mathrm{N}$ bond is formed by the $\mathrm{tBu}$ group of one monomer in a dimer (distance 2.74 $\AA$ ), being positioned above Ni atom of the pyrimidine group of one monomer in the other layer (see Figs 3 and $S_{3}$ ) The second interaction is reciprocal to this, and involve the $\mathrm{tBu}$ as well as pyrimidine groups of the same monomers not participating in the first bond. The unsubstituted $\mathrm{Cp}$ rings of the ferrocene moieties of the weakly bounded monomers are positioned on opposite sides of the overall plane of the dimer. Toluene solvent molecules fill hydrophobic voids in the crystal packing.

The structure of $\mathbf{5 m}$ has been calculated by using the density functional combination CAM-B3LYP combined with the $6-31 \mathrm{G}^{*}$ basis set and was found to agree very well with the XRD data (Figure $\mathrm{S}_{4}$ ), except the angle between the planes of cyclopentadienyl and pyrimidine rings $\left(15.5^{\circ}\right.$ for the XRD and $0.9^{\circ}$ for the optimized structure, respectively) and the position of toluene.

Although there was a good agreement between the crystallographic and NMR data of 2-ureido-4-ferrocenylpyrimidines 5, their further NMR analysis showed the existence of two different structures in solution. Two sets of signals appeared in their ${ }^{1} \mathrm{H}$ NMR spectra at lower temperature. As an example, in the spectra of $\mathbf{5 g}$ (Figure 4) different signals of the protons of the cyclopentadienyl ring (at $4.11 \mathrm{ppm}, 4.55 \mathrm{ppm}$ and $4.81 \mathrm{ppm}$ for the major component and at $4.06 \mathrm{ppm}, 4.49$ ppm and $4.89 \mathrm{ppm}$ for the minor compound), of the pyrimidine-ring proton (at $6.75 \mathrm{ppm}$ and $6.74 \mathrm{ppm}$ ) as well as of $\mathrm{NH}$ protons (at $7.63 \mathrm{ppm} / 7.32 \mathrm{ppm}$ and at $9.49 \mathrm{ppm} / 9.53$ ppm) could be distinguished at $-60{ }^{\circ} \mathrm{C}$. Because of the similar chemical shifts of $\mathrm{NH}$ protons of the two components, the existence of a monomeric and a dimeric form is not likely. (Chemical shifts of $\mathrm{NH}$ protons of $\mathrm{H}$-bonded dimers of ureido-pyrimidines are in the region of 10 to $14 \mathrm{ppm} .^{10}$ ) At the same time, compounds $\mathbf{5}$ can form different isomeric structures (Figure 5). Either of the two pyrimidine nitrogen atoms can be the acceptor of the intramolecular H-bond (A and B), and in principle, other tautomeric forms ( $\mathbf{C}$ and $\mathbf{D})$ may also exist. In order to shed light on the relative stability of these isomers, some theoretical studies were carried out. Molecular geometries and energies were calculated at the CAM$\mathrm{B}_{3} \mathrm{LYP} / 6-31 \mathrm{G}^{*}$ level for the two conformers (A and B in Figure 5) of compounds $\mathbf{5 g}, \mathbf{5 m}$ and 50 (Figure $\mathrm{S}_{5}$ ). Only a minimal difference was found between the Gibbs free energies of the two isomers (Table 5). Analogous calculations for the $\mathbf{C}$ and $\mathbf{D}$ tautomeric forms of $\mathbf{5 g}$ gave very large free energy difference ( 53.5 and $76.0 \mathrm{~kJ} / \mathrm{mol}$ respectively, with respect to conformer $\mathbf{A}$ ), so the presence of these tautomers can be excluded. The conclusion is then that the two forms present in the solution are $\mathbf{A}$ and $\mathbf{B}$ and they correspond to the two sets of signals in the NMR spectra of the compounds $\mathbf{5 g}$ (Figure 4) and $\mathbf{5 m}$ (Figure S6) at low temperature.

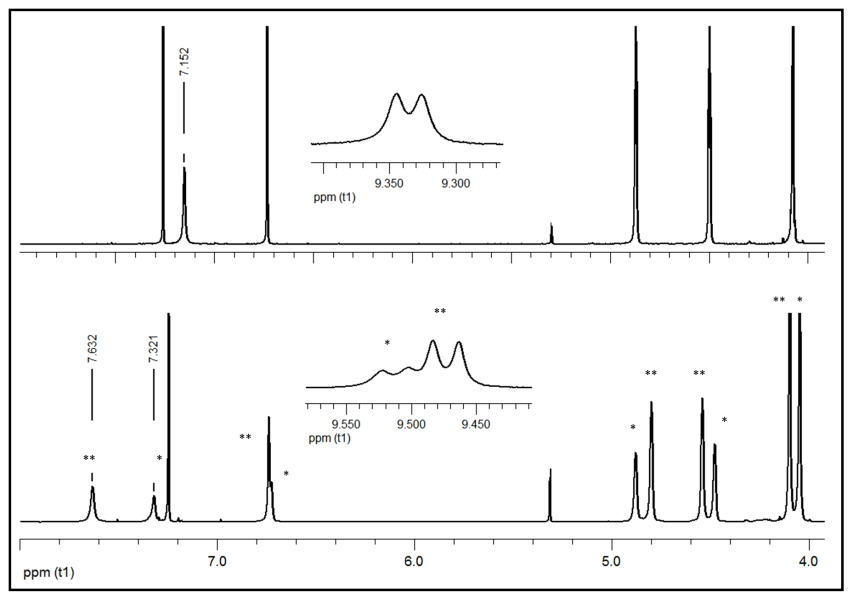

Figure 4. ${ }^{1} \mathrm{H}$ NMR spectra of $5 \mathrm{~g}$ at $27{ }^{\circ} \mathrm{C}$ (top) and $-60{ }^{\circ} \mathrm{C}$ (bottom) $\left({ }^{*}\right.$ : minor conformer, ${ }^{* *}$ : major conformer).

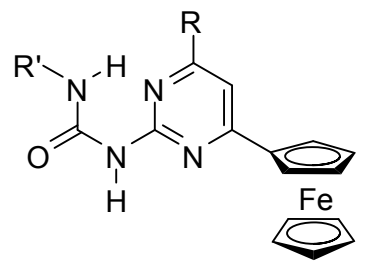

A

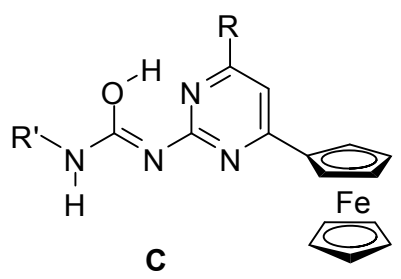<smiles>[R]c1cc(-c2ccccc2)nc(N([TlH])C(=O)N([R])NCc2ccccc2)n1</smiles>

B

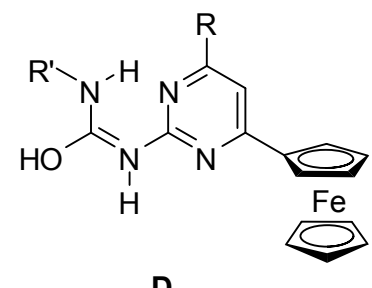

D
Figure 5. Possible isomeric structures of compounds 5 
Table 5. Calculated free energy differences between the two lowest-energy conformers of 2-ureido-4ferrocenylpyrimidine hosts $(5 \mathrm{~g}, 5 \mathrm{~m}$ and 50$)$ in the absence (B wrt. $A$ ) and in the presence (B' wrt $\left.A^{\prime}\right)$ of 2,6-diaminopyridine (guest) obtained using the CAM-B3LYP density functional with the $6-3_{1} G^{*}$ basis set

\begin{tabular}{|l|c|c|c|c|}
\hline \multirow{2}{*}{ host } & \multicolumn{2}{|c|}{$298.16 \mathrm{~K}$} & \multicolumn{2}{c|}{$213.15 \mathrm{~K}$} \\
\cline { 2 - 5 } & $\begin{array}{c}\Delta \mathrm{G}_{\mathrm{B}-\mathrm{A}} \\
(\mathrm{kJ} / \mathrm{mol})\end{array}$ & $\begin{array}{c}\Delta \mathrm{G}_{\mathrm{B}^{\prime}-\mathrm{A}^{\prime}} \\
(\mathrm{kJ} / \mathrm{mol})\end{array}$ & $\begin{array}{c}\Delta \mathrm{G}_{\mathrm{B}-\mathrm{A}} \\
(\mathrm{kJ} / \mathrm{mol})\end{array}$ & $\begin{array}{c}\Delta \mathrm{G}_{\mathrm{B}^{\prime}-\mathrm{A}^{\prime}} \\
(\mathrm{kJ} / \mathrm{mol})\end{array}$ \\
\hline $\mathbf{5 g}$ & 0.6 & 9.0 & 0.7 & 7.6 \\
\hline $\mathbf{5 m}$ & 1.8 & 7.7 & 1.2 & 7.0 \\
\hline $\mathbf{5 0}$ & 0.5 & 6.1 & 0.2 & 5.3 \\
\hline
\end{tabular}

Molecular recognition studies. Both isomers (A and $\mathbf{B}$ ) of compounds $\mathbf{5}$ might form a triplet of hydrogen bonds via an acceptor - donor - acceptor (ADA) bonding pattern. Host-guest interactions were studied using 2,6diaminopyridine (6) as the guest (Figure 6).
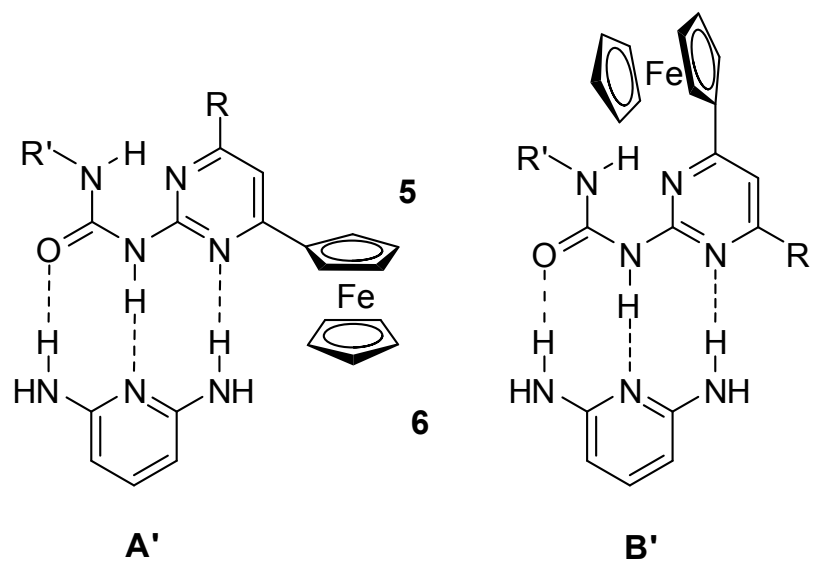

Figure 6. Host-guest interactions with 2,6-diaminopyridine

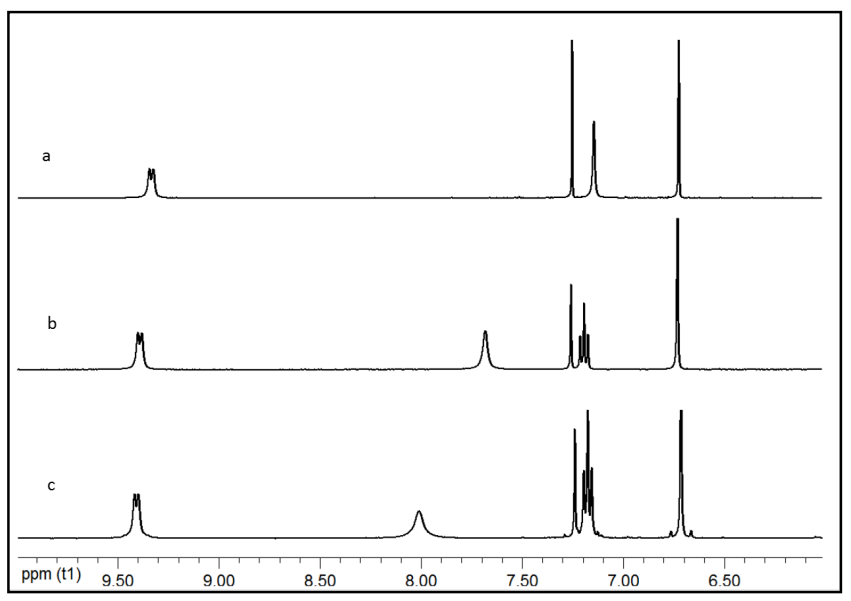

Figure 7. ${ }^{1} \mathrm{H}$ NMR spectra of $\mathbf{5 g}+\mathbf{6}$ mixtures at $27{ }^{\circ} \mathrm{C}$ (a: $\mathbf{5 g}, \mathrm{b}$ : $5 g+0.7$ eq 6 , c: $5 g+1.5$ eq 6$)$

In the ${ }^{1} \mathrm{H}$ NMR spectra of mixtures of $\mathbf{5 g}$ and 6 (Figure 7 ) the signal of the $\mathbf{N H}$ proton of $\mathbf{5 g}$ which is not involved in the internal $\mathrm{H}$-bond undergoes a downfield shift implying the formation of an intermolecular hydrogen bond with the guest 6.

Upon cooling the mixtures, two sets of signals (Figure 8) appear corresponding probably to the two isomeric structures ( $\mathbf{A}^{\prime}$ and $\mathbf{B}^{\prime}$, Figure 6 ). Comparing the spectra of pure $\mathbf{5 g}$ (Figure 4) and $\mathbf{5 g / 6}$ mixtures (Figure 8) at low temperatures, a downfield shift of $\mathrm{NH}$ signals corresponding to $\mathrm{NH}$ bonds not involved in the intramolecular $\mathrm{H}$-bonds can be noticed in case of both conformers. (At $-60{ }^{\circ} \mathrm{C}$ this signal of the major isomer overlaps with the peak of the protons in intramolecular H-bond.) It should be mentioned that according to the NMR measurements, the ratio of the two conformers changes by the addition of guest $\mathbf{6}$ in favor of the more stable form, $\mathbf{A}^{\prime}$. The difference in the integral values of peaks corresponding to the two isomers becomes greater when the ratio of 6/5g increases (Figure 9) indicating that the system is pushed towards the formation of host-guest complexes. This observation is in good agreement with the results of the computational studies that show that the free energy difference between the isomeric adducts favoring $\mathbf{A}^{\prime}$ is significantly larger than that between conformers $\mathbf{A}$ and $\mathbf{B}$ of $\mathbf{5 g}$ (Table 5).

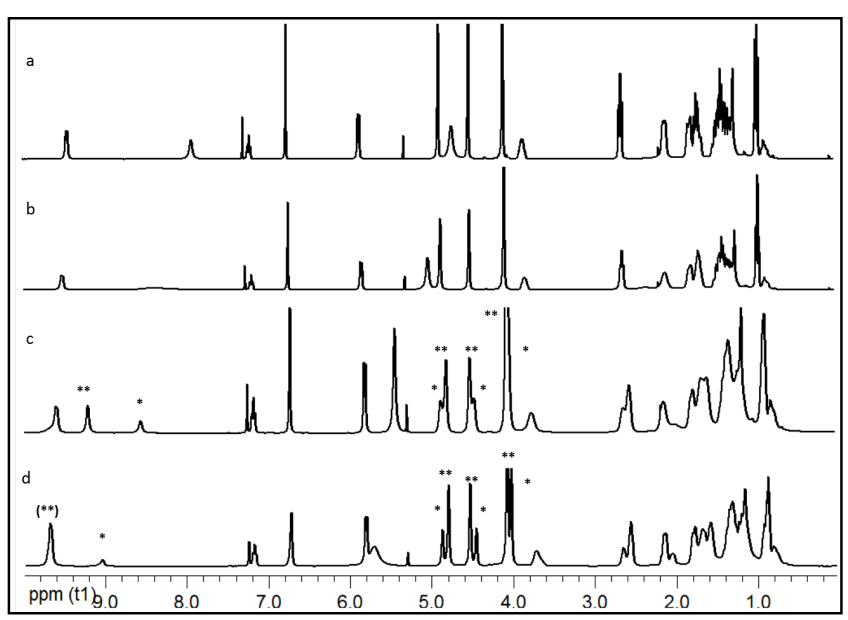

Figure 8. ${ }^{1} \mathrm{H}$ NMR spectra of the $\mathbf{5 g}+0.5$ eq 6 mixture at a: 20 ${ }^{\circ} \mathrm{C}$, b: o ${ }^{\circ} \mathrm{C}$, c: $-30{ }^{\circ} \mathrm{C}$, d: $-60{ }^{\circ} \mathrm{C}\left({ }^{*}\right.$ : minor isomer, ${ }^{* *}$ : major isomer)

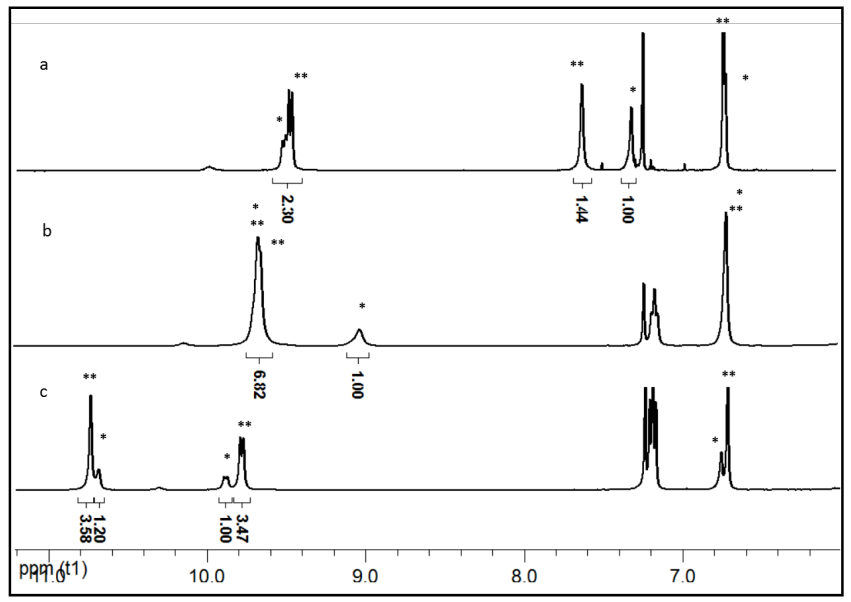


Figure 9. ${ }^{1} \mathrm{H}$ NMR spectra of $\mathbf{5 g}+\mathbf{6}$ mixtures at $-60{ }^{\circ} \mathrm{C}$ (a: $5 \mathrm{~g}$, b: $5 \mathrm{~g}+0.5$ eq 6 , c: $5 \mathrm{~g}+1.5$ eq 6$)\left({ }^{*}\right.$ : minor isomer, ${ }^{* *}$ : major isomer)

Cyclic voltammogram of $\mathbf{5 g}$ in $\mathrm{CH}_{3} \mathrm{CN}$ shows well-defined and reproducible anodic and cathodic peaks related to the $\mathrm{Fc} / \mathrm{Fc}^{+}$redox couple with quasireversible behavior (Figure $\mathrm{S}_{28}$ ). The value of the peak separation potential is $\Delta \mathrm{E}_{\mathrm{p}}=$ $\left(E_{p a}-E_{p c}\right)=72 \mathrm{mV}$, greater than $59 \mathrm{mV}$ expected for a reversible system.

Cyclic voltammetry studies also support the formation of host-guest complexes in the case of compound $5 \mathrm{~g}$.Titration experiments involving the addition of guest 6 to a $0.5 \mathrm{mM}$ solution of host $5 \mathrm{~g}$ in $\mathrm{CH}_{2} \mathrm{Cl}_{2}$ results in an anodic shift of the $E_{p a}$ value (Figure 10). This observation indicates that the formation of H-bonds between host $\mathbf{5 g}$ and guest $\mathbf{6}$ in close proximity to the ferrocenyl moiety hinders its oxidation to a ferrocenium cation. At increased guest concentrations the reduction waves flattened and the redox process became irreversible. It is probable that after the electron transfer of the oxidation, a chemical process occurs which prevents reduction from being observed (e.g., adsorption of the oxidized complex onto the working electrode surface). Similar results were obtained on two other spectral graphite electrodes (Figure S8) so the shift of the $E_{p a}$ value cannot be attributed to the electrode only.

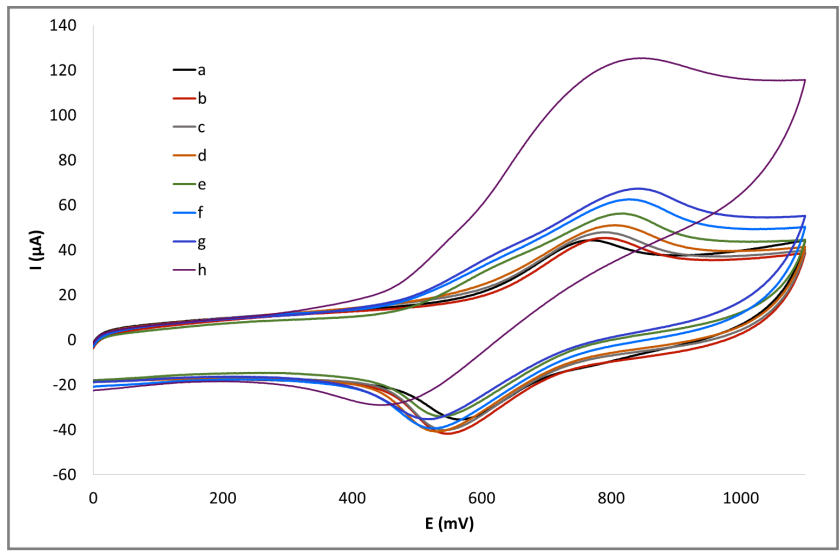

Figure 10. Cyclic voltammograms of $5 \mathrm{~g}(1 \mathrm{mM}, \mathrm{a})$ obtained upon stepwise addition of 6 (b: $0.25 \mathrm{mM}$, c: $0.5 \mathrm{mM}$, d: 0.75 mM, e: 1.o mM, f: 2.0 mM, g: $3.0 \mathrm{mM}$, h: 10.0 mM). Solvent: dichloromethane, supporting electrolyte: o.1 $\mathrm{M} \mathrm{TBAClO}_{4}$, spectral graphite electrode $(\mathrm{OD}=3 \mathrm{~mm})$ at $0.1 \mathrm{~V} / \mathrm{s}$ scan rate.

A similar anodic shift was observed in LSV experiments (Figure 11). From the shift of cathodic peak potentials an association constant with $\log \mathrm{K}(\mathrm{obs})=3.40$ could be calculated by the Benesi Hildebrand method (Figure S9).

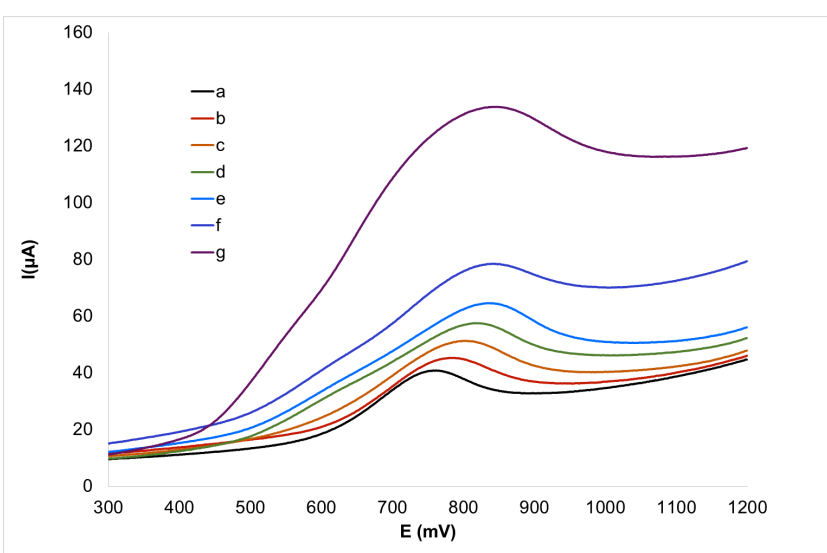

rigure 11. LDV response or $\mathbf{5 g}(0.5 \mathrm{mIvl}$, a) upon stepwise addition of 6 (b: $0.125 \mathrm{mM}$, c: $0.375 \mathrm{mM}$, d: $0.5 \mathrm{mM}$, e: 1.0 $\mathrm{mM}$, f: $1.5 \mathrm{mM}$, g: 5.0 mM). Solvent: dichloromethane, supporting electrolyte: o.1 $\mathrm{M} \mathrm{TBAClO}_{4}$, spectral graphite electrode $(\mathrm{OD}=3 \mathrm{~mm})$ at $0.1 \mathrm{~V} / \mathrm{s}$ scan rate.

The difference in the $E_{p a}$ value of $\mathbf{5 g}$ and a mixture of 5g/6=1/1 observed in $\mathrm{CH}_{2} \mathrm{Cl}_{2}$ (Figure 10, Figure S8) diminishes in solvents that contains increasing amounts of $\mathrm{CH}_{3} \mathrm{CN}$ (Table $\mathrm{S} 1$, Figure 12). This shows that the presence of the more polar $\mathrm{CH}_{3} \mathrm{CN}$ decreases the stability of the adduct. The shift of the oxidation wave of the $\mathbf{5 g} / \mathbf{6}$ mixture to smaller potentials in the presence of $\mathrm{CH}_{3} \mathrm{CN}$ supports the disruption of the $\mathrm{H}$-bonds pushing the system towards the more readily oxidizable non-complexed form.

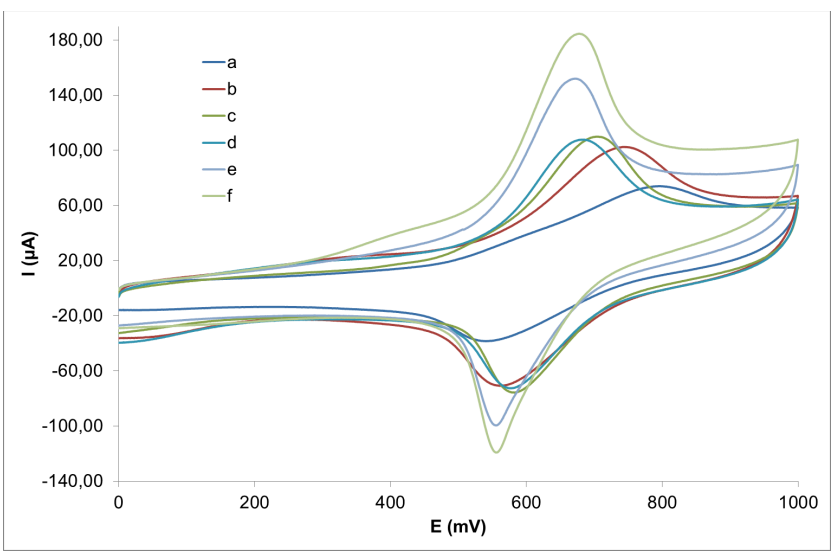

Figure 12. Cyclic voltammograms of $\mathbf{5 g + 6}$ (concentrations: 1 $\mathrm{mM}, 1 \mathrm{mM})$ in $\mathrm{CH}_{2} \mathrm{Cl}_{2} / \mathrm{CH}_{3} \mathrm{CN}$ solvent mixtures. $\left(\mathrm{CH}_{2} \mathrm{Cl}_{2} / \mathrm{CH}_{3} \mathrm{CN}\right.$ ratios: a: $1 / 0$; b: $5 / 1 ; \mathrm{c}: 2 / 1 ; \mathrm{d}: 1 / 1$; e: $1 / 2 ; \mathrm{f}: 1 / 5$. Supporting electrolyte: o.1 $\mathrm{M} \mathrm{TBAClO}_{4}$, spectral graphite electrode $(\mathrm{OD}=3 \mathrm{~mm})$ at $0.1 \mathrm{~V} / \mathrm{s}$ scan rate.

\section{CONCLUSIONS}

New 2-ureido-4-ferrocenyl pyrimidine derivatives (5a-p) were obtained in good yields in a three-step reaction sequence involving carbonylative Sonogashira coupling of iodoferrocene followed by addition/cyclocondensation with guanidine carbonate and acylation of the amino group with alkyl/aryl isocyanates.

In solution, the existence of two conformers of the products has been observed by NMR investigations. Density functional theory calculations support these experimental data showing only a small difference in the stability of the two 
isomers. The solid-state structure of the more stable conformer was also confirmed by X-ray crystallography pointing to the presence of an intramolecular hydrogen bond between a nitrogen atom of the pyrimidine ring and an $\mathrm{NH}$ group of the urea moiety.

The products may serve as hosts for appropriate molecular guests with a donor - acceptor - donor H-bonding pattern. Complexation with 2,6-diaminopyridine (6) results in a change in the ${ }^{1} \mathrm{H}$ NMR spectra and also in the electrochemical properties of the 2-ureido-4-ferrocenyl pyrimidines. In the presence of guest 6 an anodic shift of $E_{p a}$ values can be observed showing the preference of the non-oxidized form of the host $\mathbf{5 g}$ towards the guest 6 .

\section{ASSOCIATED CONTENT}

\section{Supporting Information}

Supporting information contains figures showing NMR investigation supporting the conclusions of the manuscript, crystal packing of compound $\mathbf{5 g}$, some computed structures, results of electrochemical measurements and NMR spectra of all of the new compounds (pdf). Crystallographic data of $\mathbf{5 m}$ (cif).A text file of all computed molecule Cartesian coordinates in .xyz format for convenient visualization.

\section{AUTHOR INFORMATION}

\section{Corresponding Author}

E-mail:skodane@almos.uni-pannon.hu

\section{Notes}

The authors declare no competing financial interests.

\section{ACKNOWLEDGMENT}

The support of the National Research, Development and Innovation Office (OTKA K105632 and OTKA K108966) is acknowledged. L. Nagy's work was supported by the PTE ${ }_{3} \mathrm{D}$ Project, GINOP 2.3.2-15-2016-00022, as part of the Economic Development and Innovation Operational Program (GINOP).

\section{REFERENCES}

(1) Chaubey, A.; Malhotra, B. D. Biosens. Bioelectron. 2002, 17, 441-456.

(2) Molina, P.; Tárraga, A.; Caballero, A. Eur. J. Inorg. Chem. 2008, 3401-3417.

(3) Alonso, B.; Casado, C. M., Cuadrado, I.; Moran, M.; Kaifer, A. E. Chem. Commun. 2002, 1778-1779.

(4) (a) Laurent, P.; Miyaji, H.; Collinson, S. R.; Prokeš, I.; Moody, C. J.; Tucker, J. H. R.; Slawin, A. M. Z. Org. Lett., 2002, 4, 4037-4040. (b) Willener, Y.; Joly, Y. K. M.; Moody, C. J.; Tucker, J. H. R. J. Org. Chem. 2oo8, 73, 1225-1233. (c) Mulas, A.; Willener, Y.; Carr-Smith, J.; Joly, K. M.; Male, L.; Moody, C. J.; Horswell, S. L.; Nguyen, H. V.; Tucker, J. H. R. Dalton Trans., 2015, 44, 7268-7275.

(5) Otón, F.; Tárraga, A.; Velasco, M. D.; Molina, P. Dalton Trans. 2006, 3685-3692.

(6) Solařová, H.; Císařová, I.; Štěpnička, P. Organometallics 2014, 33, 4131-4147.

(7) Moon, K.; Kaifer,A. E. J. Am. Chem. Soc. 2oo4, 126, 15016-15017

(8) Yang, L.; Tan, X.; Wang, Z.; Zhang, X. Chem. Rev. 2o15, 115, 7196-7239.
(9) Yamanaka, M. J. Incl. Phenom. Macrocycl. Chem. 2013, 77, 33-48.

(10) Beijer, F. H.; Sijbesma, R. P.; Kooijman, H.; Spek, A. L.; Meijer, E. W. J. Am. Chem. Soc. 1998, 120, 6761-6769.

(11) Zou, S.; Zhang,Z.; Förch, R.; Knoll, W.; Schönherr, H.; Vancso, G. J. Langmuir 2003, 19, 8618-8621.

(12) Moon, K.; Philip, I.; Sun, H.; Kaifer, A. E. J. Solid State Electrochem. 2007, 11, 1635-1641.

(13) Alexander, A. M.; Bria, M.; Brunklaus, G.; Caldwell, S.; Cooke, G.; Garety, J. F.; Hewage, S. G.; Hocquel, Y.; McDonald, N.; Rabani, G.; Rosair, G.; Smith, B. O.; Spiess, H. W.; Rotello, W. M.; Woisel, P. Chem. Commun. 2007, 2246-2248.

(14) Feng, K.; Yu, M. L.; Wang, S. M.; Wang, G. X.; Tung C. H., Wu, L. Z. ChemPhysChem 2013, 14, 198 - 203.

(15) Sun, H.; Steeb, J.; Kaifer, A. E. J. Am. Chem. Soc. 20o6, 128, 2820-2821.

(16) Wang, S. M.; Zhao, R. N.; Wang, Q. G.; Guo, H.; Li, J. H.; Zhang, W. H. Acta Phys. -Chim. Sin. 2016, 32, 611616.

(17) Cooke, C.; de Cremiers, H. A.; Duclairoir, F. M. A.; Leonardi, J.; Rosaira, G.; Rotello, V. M. Tetrahedron 2003, 59, 3341-3347.

(18) (a) Fehér, C.; Kuik, A.; Márk, L.; Kollár, L.; SkodaFöldes, R. J. Organomet. Chem. 2oo9, 694, 4036-4041. (b) Fehér, C.; Habuš, I.; Wouters, J.; Skoda-Földes, R. Monats. Chem. 2014, 145, 1981-1986.

(19) (a) Rausch, M. D.; Ciappenelli, D. J. J. Organomet. Chem. 1967, 10, 127-136, (b) Watanabe, M.; Araki, S.; Butsugan, Y. J. Org. Chem. 1991, 56, 2218-2224.

(20) Urbán, B.; Srankó, D.; Sáfrán, G.; Ürge, L.; Darvas, F.; Bakos, J.; Skoda-Földes, R. J. Mol. Catal. A 2014, 395, 364-372.

(21) Plażuk, D.; Zakrzewski, J. J. Organomet. Chem. 2009, 694, 1802-1806.

(22) Sheldrick, A. Acta Cryst. 2oo8, A64, 112-22

(23) Speck, A.L. PLATON, A Multipurpose Crystallographic Tool; Utrecht University, Utrecht (The Netherlands), 2005.

(24) Frisch, M. J.; Trucks, G. W.; Schlegel, H. B.; Scuseria, G. E.; Robb, M. A.; Cheeseman, J. R.; Scalmani, G.; Barone, V.; Mennucci, B.; Petersson, G. A.; Nakatsuji, H.; Caricato, M.; Li, X.; Hratchian, H. P.; Izmaylov, A. F.; Bloino, J.; Zheng, G.; Sonnenberg, J. L.; Hada, M.; Ehara, M.; Toyota, K.; Fukuda, R.; Hasegawa, J.; Ishida, M.; Nakajima, T.; Honda, Y.; Kitao, O.; Nakai, H.; Vreven, T.; Montgomery, J. A., Jr.; Peralta, J. E.; Ogliaro, F.; Bearpark, M.; Heyd, J. J.; Brothers, E.; Kudin, K. N.; Staroverov, V. N.; Kobayashi, R.; Normand, J.; Raghavachari, K.; Rendell, A.; Burant, J. C.; Iyengar, S. S.; Tomasi, J.; Cossi, M.; Rega, N.; Millam, N. J.; Klene, M.; Knox, J. E.; Cross, J. B.; Bakken, V.; Adamo, C.; Jaramillo, J.; Gomperts, R.; Stratmann, R. E.; Yazyev, O.; Austin, A. J.; Cammi, R.; Pomelli, C.; Ochterski, J. W.; Martin, R. L.; Morokuma, K.; Zakrzewski, V. G.; Voth, G. A.; Salvador, P.; Dannenberg, J. J.; Dapprich, S.; Daniels, A. D.; Farkas, Ö.; Foresman, J. B.; Ortiz, J. V.; Cioslowski, J.; Fox, D. J. Gaussian o9, Revision A.02, Gaussian, Inc., Wallingford, CT, 2009.

(25) Yanai, T.; Tew, D. P.; Handy, N. C. Chem. Phys. Lett. 2004, 393, 51-57.

(26) Hariharan, P. C.; Pople, J. A. Mol. Phys. 1974, 27, 209-214.

(27) Schaftenaar, G.; Noordik, J. H. J. Comput.-Aided Mol. Design, 2000, 14, 123-134. 
(28) Vanmaele, L.; Loccufier, J.; Meijer, E.; Janssen, H.; Fransen, P.; EP 1486 539, 2004. 


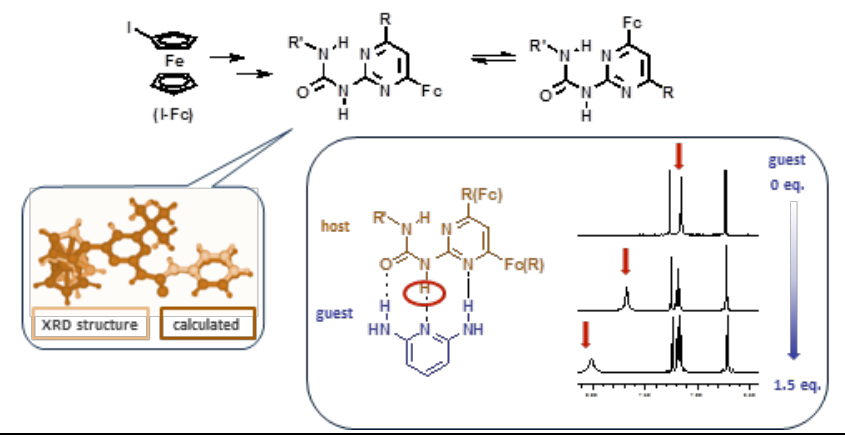

